Y. Murata

Nagoya Math. J.

Vol. 139 (1995), 37-65

\title{
CLASSICAL SOLUTIONS OF THE THIRD PAINLEVÉ EQUATION
}

\author{
YOSHIHIRO MURATA
}

\section{Introduction and main results}

The big problem "Do Painlevé equations define new functions?", what is called the problem of irreducibilities of Painlevé equations, was essentially solved by H. Umemura [16], [17] and K. Nishioka [9].

Umemura [16] analyzed Painlevé's Stockholm Lessons [15] and extracted the concept of "classical functions". To define "classical functions", Umemura introduced the permissible operations to construct new known functions from already known functions. First, we note that we identify a holomorphic function $f$ on an open set $U \subset \mathbf{C}$ with its restriction $\left.f\right|_{V}$ onto an open subset $V \subset U$. Let $S$ be a certain set of meromorphic functions on a domain $D \subset \mathbf{C}$. We assume that all the elements in $S$ are already known functions. Permissible operations to construct new known functions from the set $S$ are as follows.

Definition I [16, Part II §2]. (0) Let $f(t) \in S$. Then the derived function $f^{\prime}(t)$ is a new known function.

(P1) If $f_{1}, f_{2} \in S$, then the sum $f_{1}+f_{2}$ and the product $f_{1} f_{2}$ are new known functions. Moreover if $f_{2} \neq 0$, then the quotient $f_{1} / f_{2}$ is a new known function.

(P2) Let $a_{1}, \cdots, a_{n} \in S$. Then any solution $f$ of an algebraic equation $f^{n}+$ $a_{1} f^{n-1}+\cdots+a_{n}=0$ is a new known function.

(P3) Let $f(t) \in S$. Then the quadrature $\int f(t) d t$ is a new known function.

(P4) Let $a_{1}, \cdots, a_{n} \in S$. Then any solution $f$ of a linear differential equation $d^{n} f / d t^{n}+a_{1} d^{n-1} f / d t^{n-1}+\cdots+a_{n} f=0$ is a new known function.

(P5) Let $\Gamma \subset \mathbf{C}^{n}$ be a lattice such that the quotient $\mathbf{C}^{n} / \Gamma$ is an abelian variety. Let $\pi: \mathbf{C}^{n} \rightarrow \mathbf{C}^{n} / \Gamma$ be the projection. Let $f_{1}, \cdots, f_{n} \in S$ be holomorphic functions on a domain $D \subset \mathbf{C}$ and $\phi$ be a meromorphic function on $\mathbf{C}^{n} / \Gamma$. Then the function $\phi \cdot \pi \cdot\left(f_{1}, \cdots, f_{n}\right)$ is a new known function if it is not the constant function taking

Received March 28, 1994. 
infinity.

In any operation of (P2), $\cdots$, (P5), we consider that we take an appropriate subdomain $D^{\prime} \subset D$ such that the newly constructed function is meromorphic and single valued on $D^{\prime}$. Using these permissible operations, Umemura defined "classical functions" as follows:

Definition II [16, Part II $\S 2$, Definition (2.27)]. Let $f$ be a meromorphic function on a domain $D \subset \mathbf{C}, M_{D}$ be the set of all meromorphic functions on $D$ and $\mathbf{C}(t)$ be the field of rational functions in a variable $t$. The function $f$ is called classical if and only if there exists a tower of differential subfields $K_{0}=\mathbf{C}(t)$, $K_{1}, \cdots, K_{m}$ of $\mu_{D}$ such that

(i) For any $j=1, \cdots, m, K_{j}=K_{j-1}\left\langle g_{j}\right\rangle=K_{j-1}\left(g_{j}, g_{j}{ }^{\prime}, g_{j}^{\prime \prime}, \cdots\right)$, where $g_{j}$ is a meromorphic function obtained by one of permissible operations (P2), $\cdots$, (P5) from the field $K_{j-1}$.

(ii) $f \in K_{m}$.

In this sense, rational functions in one variable, $e^{t}, \log t$, elliptic functions, the hypergeometric function and confluent hypergeometric functions are examples of classical functions. It is non-classical functions that are essentially new functions. We call a non-classical function an irreducible function.

Using the idea of Nishioka [9] and the fact that Painlevé I does not have algebraic solutions, Umemura [17] showed the theorem that every solution of Painlevé I is irreducible. After that, by the same idea, M. Noumi [10] clarified the distribution of classical solutions and irreducible solutions of Painleve II, K. Okamoto [14] solved the case of Painlevé IV. They slightly generalized the techniques of Umemura [17] and used the facts on rational solutions of Painleve II and IV and on solutions of Riccati equations contained in Painlevé II and IV [7]. Then our next target is Painleve III. In this paper, we investigate the distribution of classical solutions of Painlevé III', which is equivalent to Painlevé III, in connection with the transformation group of solutions. Particularly, we completely determine all algebraic solutions. In the forthcoming paper [8], we prove a theorem that except

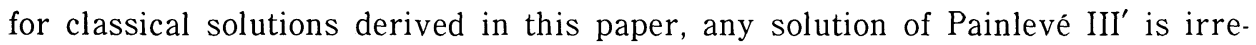
ducible.

\subsection{Two expressions of the third Painlevé equation}

Painlevé III has an equivalent equation Painlevé III' [13, Introduction]; 


$$
\begin{gathered}
P_{\mathrm{III}}: \frac{d^{2} y}{d x^{2}}=\frac{1}{y}\left(\frac{d y}{d x}\right)^{2}-\frac{1}{x} \frac{d y}{d x}+\frac{1}{x}\left(\alpha y^{2}+\beta\right)+\gamma y^{3}+\frac{\delta}{y} \\
P_{\mathrm{III}}: \frac{d^{2} q}{d t^{2}}=\frac{1}{q}\left(\frac{d q}{d t}\right)^{2}-\frac{1}{t} \frac{d q}{d t}+\frac{q^{2}}{4 t^{2}}(\gamma q+\alpha)+\frac{\beta}{4 t}+\frac{\delta}{4 q} .
\end{gathered}
$$

In fact, a solution of $P_{\text {III }}$ corresponds to that of $P_{\text {III' }}$ by the change of the variables:

$$
t=x^{2}, \quad q=x y .
$$

In this paper, we mainly treat $P_{\mathrm{III}}$, because it has a transformation group with good structure (See 1.2).

As is well known [13, Proposition 1.1], by the change of the variables: $t=t_{1}$, $q=t / q_{1}, P_{\mathrm{III}}(\alpha, \beta, \gamma, \delta)$ is transformed into $P_{\mathrm{III}}(-\beta,-\alpha,-\delta,-\gamma)$. In the same way, by the change of the variables: $t=t_{1}{ }^{2}, q=q_{1}{ }^{2}, P_{\mathrm{III}}(\alpha, \beta, 0,0)$ is transformed into $P_{\mathrm{III}}(0,0,2 \alpha, 2 \beta)$ [11 II, Remark 1]. The correspondence (1.3) implies that $P_{\text {III }}(\alpha, \beta, \gamma, \delta)$ also has similar transformations to the above ones.

From these facts, we may consider that the values of the complex parameters $\alpha, \beta, \gamma, \delta$ of $P_{\mathrm{III}}$ and $P_{\mathrm{III}}$ satisfy one of the three cases:
(A) $\alpha=\gamma=0$ (or $\beta=\delta=0$ )
(B) $\gamma=0, \alpha \delta \neq 0$ (or $\delta=0, \beta \gamma \neq 0$ )
(C) $\gamma \delta \neq 0$.

In the case (A), $P_{\text {III }}$ and $P_{\text {III }}$ are solvable by quadratures [13, Proposition 1.5]. In the case (B), V. I. Gromak [1], [4, Theorem 2], [5, 2] showed that $P_{\text {III }}$ has 3 -sheeted algebraic solutions for special values of $\beta$ (or $\alpha$ ). In the case (C), any solution of $P_{\mathrm{III}}$ (resp. $P_{\mathrm{III}}$ ) governs the isomonodromic deformation of a second order linear differential equation $L_{\mathrm{III}^{\prime}}$ (resp. $L_{\mathrm{III}}$ ) which has irregular singularities of Poincaré rank 1 at the origin and at infinity, and a nonlogarithmic singularity at $q$ [11 II, Proposition 1], [12, 4.3]. Then the case $(\mathrm{C})$ is essential for $P_{\mathrm{III}}$ and $P_{\text {III }}$. In this case, Gromak [5, Theorem 9] obtained the necessary and sufficient conditions for $P_{\text {III }}(\alpha, \beta, 1,-1)$ to have rational solutions by the use of transformations of solutions of $P_{\mathrm{III}}$. But, as we will mention in 1.2 , the transformation group of solutions of $P_{\mathrm{III}}$ is isomorphic to the Affine Weyl group of the type $B_{2}$, and so, we can treat $P_{\mathrm{III}}$ more successfully than $P_{\mathrm{III}}$. Therefore, in this paper, by the help of this transformation group of $P_{\mathrm{III}}$ and by different approaches from those of Gromak [5], in this case (C), we investigate algebraic solutions and solutions expressible by Riccati solutions of $P_{\mathrm{III}^{\prime}}$ in detail. In the process of studying 
algebraic solutions of $P_{\mathrm{III}}$, we obtain a result on algebraic solutions of $P_{\mathrm{III}}$ which contains the theorem by Gromak [5, Theorem 9].

Let parameters $(\alpha, \beta, \gamma, \delta)$ satisfy the case $(\mathrm{C})$. Then $(\alpha, \beta, \gamma, \delta)$ can be replaced by other parameters $\left(\eta_{0}, \eta_{\infty}, \theta_{0}, \theta_{\infty}\right)[11 \mathrm{II}, 2]$;

$$
\alpha=-4 \eta_{\infty} \theta_{\infty}, \beta=4 \eta_{0}\left(\theta_{0}+1\right), \gamma=4 \eta_{\infty}{ }^{2}, \delta=-4 \eta_{0}^{2} \text {. }
$$

In addition, by the change of variables:

$$
t=\lambda t_{1}, \quad q=\mu q_{1}, \quad(\lambda \mu \neq 0),
$$

$P_{\mathrm{III}}\left(\eta_{0}, \eta_{\infty}, \theta_{0}, \theta_{\infty}\right)$ is transformed into $P_{\mathrm{III}}\left((\lambda / \mu) \eta_{0}, \mu \eta_{\infty}, \theta_{0}, \theta_{\infty}\right)$. Then, if the values of $\lambda$ and $\mu$ are chosen appropriately, $P_{\mathrm{III}}\left(\eta_{0}, \eta_{\infty}, \theta_{0}, \theta_{\infty}\right)$ is transformed into a canonical type equation $P_{\mathrm{III}}\left(1,1, \theta_{0}, \theta_{\infty}\right)$, which we express by $P_{\mathrm{III}}\left(\theta_{0}\right.$, $\left.\theta_{\infty}\right)$. From now on, we mainly consider this canonical type equation

$$
P_{\mathrm{III}}\left(\theta_{0}, \theta_{\infty}\right): \frac{d^{2} q}{d t^{2}}=\frac{1}{q}\left(\frac{d q}{d t}\right)^{2}-\frac{1}{t} \frac{d q}{d t}+\frac{q^{2}}{t^{2}}\left(q-\theta_{\infty}\right)+\frac{\theta_{0}+1}{t}-\frac{1}{q} .
$$

$P_{\mathrm{III}}\left(\eta_{0}, \eta_{\infty}, \theta_{0}, \theta_{\infty}\right)$ is also transformed into a canonical type equation

$$
P_{\mathrm{III}}\left(\theta_{0}, \theta_{\infty}\right): \frac{d^{2} y}{d x^{2}}=\frac{1}{y}\left(\frac{d y}{d x}\right)^{2}-\frac{1}{x} \frac{d y}{d x}+\frac{4}{x}\left(-\theta_{\infty} y^{2}+\theta_{0}+1\right)+4 y^{3}-\frac{4}{y}
$$

by a similar transformation to (1.6).

\subsection{Transformation groups of $P_{\mathrm{III}}$ and $P_{\mathrm{III}}$}

As Okamoto [13, Introduction] pointed out, $P_{\mathrm{III}}\left(\eta_{0}, \eta_{\infty}, \theta_{0}, \theta_{\infty}\right)$ is transformed into a Hamiltonian system. In fact, putting

$$
\left\{\begin{array}{l}
q=q \\
p=\frac{t d q / d t+\eta_{\infty} q^{2}+\theta_{0} q-\eta_{0} t}{2 q^{2}},
\end{array}\right.
$$

then we get a Hamiltonian system

$$
\left\{\begin{array}{l}
\frac{d q}{d t}=\frac{1}{t}\left[2 q^{2} p-\left(\eta_{\infty} q^{2}+\theta_{0} q-\eta_{0} t\right)\right]=\frac{\partial H}{\partial p} \\
\frac{d p}{d t}=-\frac{1}{t}\left[2 q p^{2}-\left(2 \eta_{\infty} q+\theta_{0}\right) p+\frac{1}{2} \eta_{\infty}\left(\theta_{0}+\theta_{\infty}\right)\right]=-\frac{\partial H}{\partial q},
\end{array}\right.
$$

where $\quad H=(1 / t)\left[q^{2} p^{2}-\left(\eta_{\infty} q^{2}+\theta_{0} q-\eta_{0} t\right) p+(1 / 2) \eta_{\infty}\left(\theta_{0}+\theta_{\infty}\right) q\right]$. Let $H_{\mathrm{III}}\left(\theta_{0}, \theta_{\infty}\right)$ denote the Hamiltonian system which corresponds to $P_{\mathrm{III}}\left(\theta_{0}, \theta_{\infty}\right)$, i.e., 
the system (1.8) with parameters $\left(1,1, \theta_{0}, \theta_{\infty}\right)$. Okamoto obtained a transformation group $\mathbf{G}_{H}^{\prime}$ of $H_{\mathrm{III}}\left(\theta_{0}, \theta_{\infty}\right)$ and showed that it is isomorphic to the Affine Weyl group of the type $B_{2}[13$, Theorem 1]. In this paper, we consider the transformation group $\mathbf{G}^{\prime}$ of $P_{\mathrm{III}}\left(\theta_{0}, \theta_{\infty}\right)$ which is derived from $\mathbf{G}_{H}^{\prime}$ by (1.7).

Generators of $\mathbf{G}^{\prime}$ and transformations which we use later are as follows:

$$
\begin{aligned}
& s_{0} \quad\left\{\begin{array}{l}
(t, q) \rightarrow(s, Q)=(t,-t / q) \\
\left(\theta_{0}, \theta_{\infty}\right) \rightarrow\left(\bar{\theta}_{0}, \bar{\theta}_{\infty}\right)=\left(-\theta_{\infty}-1,-\theta_{0}-1\right)
\end{array}\right. \\
& s_{1}\left\{\begin{array}{l}
(t, q) \rightarrow(s, Q)=\left(t, q \frac{t d q / d t-q^{2}+\theta_{\infty} q-t}{t d q / d t-q^{2}+\theta_{0} q-t}\right) \\
\left(\theta_{0}, \theta_{\infty}\right) \rightarrow\left(\bar{\theta}_{0}, \bar{\theta}_{\infty}\right)=\left(\theta_{\infty}, \theta_{0}\right)
\end{array}\right. \\
& s_{2} \quad\left\{\begin{array}{l}
(t, q) \rightarrow(s, Q)=(-t,-q) \\
\left(\theta_{0}, \theta_{\infty}\right) \rightarrow\left(\bar{\theta}_{0}, \bar{\theta}_{\infty}\right)=\left(\theta_{0},-\theta_{\infty}\right)
\end{array}\right. \\
& l\left\{\begin{array}{l}
(t, q) \rightarrow(s, Q)=\left(t,-\frac{t}{q} \frac{t d q / d t-q^{2}-\left(\theta_{0}+2\right) q+t}{t d q / d t-q^{2}+\theta_{\infty} q+t}\right) \\
\left(\theta_{0}, \theta_{\infty}\right) \rightarrow\left(\bar{\theta}_{0}, \bar{\theta}_{\infty}\right)=\left(\theta_{0}+1, \theta_{\infty}+1\right)
\end{array}\right. \\
& l^{-1}\left\{\begin{array}{l}
(t, q) \rightarrow(s, Q)=\left(t,-\frac{t}{q} \frac{t d q / d t+q^{2}+\theta_{0} q-t}{t d q / d t+q^{2}-\theta_{\infty} q-t}\right) \\
\left(\theta_{0}, \theta_{\infty}\right) \rightarrow\left(\bar{\theta}_{0}, \bar{\theta}_{\infty}\right)=\left(\theta_{0}-1, \theta_{\infty}-1\right)
\end{array}\right. \\
& m\left\{\begin{array}{l}
(t, q) \rightarrow(s, Q)=\left(t, \frac{t}{q} \frac{t d q / d t+q^{2}-\left(\theta_{0}+2\right) q+t}{t d q / d t+q^{2}-\theta_{\infty} q+t}\right) \\
\left(\theta_{0}, \theta_{\infty}\right) \rightarrow\left(\bar{\theta}_{0}, \bar{\theta}_{\infty}\right)=\left(\theta_{0}+1, \theta_{\infty}-1\right)
\end{array}\right. \\
& m^{-1}\left\{\begin{array}{l}
(t, q) \rightarrow(s, Q)=\left(t, \frac{t}{q} \frac{t d q / d t-q^{2}+\theta_{0} q-t}{t d q / d t-q^{2}+\theta_{\infty} q-t}\right) \\
\left(\theta_{0}, \theta_{\infty}\right) \rightarrow\left(\bar{\theta}_{0}, \bar{\theta}_{\infty}\right)=\left(\theta_{0}-1, \theta_{\infty}+1\right)
\end{array}\right. \\
& h \quad\left\{\begin{array}{l}
(t, q) \rightarrow(s, Q)=(t,-q) \\
\left(\theta_{0}, \theta_{\infty}\right) \rightarrow\left(\bar{\theta}_{0}, \bar{\theta}_{\infty}\right)=\left(-\theta_{0}-2,-\theta_{\infty}\right) .
\end{array}\right.
\end{aligned}
$$

Each transformation of the above all is applicable only when a solution $q(t)$ does not vanish the numerator and the denominator of $Q . \mathbf{G}^{\prime}$ is generated by $\boldsymbol{s}_{j}$ 's 
$(j=0,1,2)$. These transformations combine together like

$$
\begin{aligned}
& s_{j}^{2}=\mathrm{id}(j=0,1,2), \\
& l=\left(s_{1} s_{2}\right)^{2} s_{0} s_{1}, m=s_{2} \ell s_{2}, h=s_{1} m s_{0}, \\
& l m=m l, h l=l^{-1} h, h m=m^{-1} h .
\end{aligned}
$$

We also introduce the transformation group $\mathbf{G}$ of $P_{\mathrm{III}}\left(\theta_{0}, \theta_{\infty}\right)$, which is derived from $\mathbf{G}^{\prime}$ by (1.3). Let $S_{j}$ denote the corresponding transformation to $s_{j}(j=0,1)$ :

$S_{0} \quad\left\{\begin{array}{l}(x, y) \rightarrow(u, Y)=(x,-1 / y) \\ \left(\theta_{0}, \theta_{\infty}\right) \rightarrow\left(\bar{\theta}_{0}, \bar{\theta}_{\infty}\right)=\left(-\theta_{\infty}-1,-\theta_{0}-1\right)\end{array}\right.$

$S_{1}\left\{\begin{array}{l}(x, y) \rightarrow(u, Y)=\left(x, y \frac{(x / 2)(d y / d x)-x y^{2}+\left(\theta_{\infty}+1 / 2\right) y-x}{(x / 2)(d y / d x)-x y^{2}+\left(\theta_{0}+1 / 2\right) y-x}\right) \\ \left(\theta_{0}, \theta_{\infty}\right) \rightarrow\left(\bar{\theta}_{0}, \bar{\theta}_{\infty}\right)=\left(\theta_{\infty}, \theta_{0}\right) .\end{array}\right.$

$S_{1}$ is applicable under the conditions $(x / 2)(d y / d x)-x y^{2}+\left(\theta_{\infty}+1 / 2\right) y-x$ $\neq 0$ and $(x / 2)(d y / d x)-x y^{2}+\left(\theta_{\infty}+1 / 2\right) y-x \neq 0$. The transformation $s_{2}$ corresponds to the transformation

$S_{\xi} \quad\left\{\begin{array}{l}(x, y) \rightarrow(u, Y)=(\xi x, \xi y) \\ \left(\theta_{0}, \theta_{\infty}\right) \rightarrow\left(\bar{\theta}_{0}, \bar{\theta}_{\infty}\right)=\left(\theta_{0},-\theta_{\infty}\right),\end{array}\right.$

where $\xi$ is $i$ or $-i(i=\sqrt{-1}) . S_{0}, S_{1}$ and $S_{\xi}$ generate $\mathbf{G}^{\prime}$. Correctly speaking, $\mathbf{G}^{\prime}$ $=\left\langle S_{0}, S_{1}, S_{i}\right\rangle=\left\langle S_{0}, S_{1}, S_{-i}\right\rangle$ holds, because $\left(S_{ \pm i}\right)^{3}=S_{( \pm i)^{3}}=S_{\mp i}$. Here, we have $S_{0}{ }^{2}=S_{1}{ }^{2}=$ id and $S_{i} S_{-i}=S_{-i} S_{i}=\mathrm{id}$. The transformation $s_{j}(j=0,1)$ actually corresponds to both of $S_{j}$ and $\left(S_{\xi}\right)^{2} S_{j}$. The transformation $m$ corresponds to the transformation

$M\left\{\begin{array}{l}(x, y) \rightarrow(u, Y)=\left(x, \frac{1}{y} \frac{(x / 2)(d y / d x)+x y^{2}-\left(\theta_{0}+3 / 2\right) y+x}{(x / 2)(d y / d x)+x y^{2}-\left(\theta_{\infty}-1 / 2\right) y+x}\right) \\ \left(\theta_{0}, \theta_{\infty}\right) \rightarrow\left(\bar{\theta}_{0}, \bar{\theta}_{\infty}\right)=\left(\theta_{0}+1, \theta_{\infty}-1\right) .\end{array}\right.$

The transformation $m^{-1}$ corresponds to the transformation

$M^{-1}\left\{\begin{array}{l}(x, y) \rightarrow(u, Y)=\left(x, \frac{1}{y} \frac{(x / 2)(d y / d x)-x y^{2}+\left(\theta_{0}+1 / 2\right) x y-x}{(x / 2)(d y / d x)-x y^{2}+\left(\theta_{\infty}-1 / 2\right) x y-x}\right) \\ \left(\theta_{0}, \theta_{\infty}\right) \rightarrow\left(\bar{\theta}_{0}, \bar{\theta}_{\infty}\right)=\left(\theta_{0}-1, \theta_{\infty}+1\right) .\end{array}\right.$

Here each transformation of the above two is applicable only when a solution 
$y(x)$ does not vanish the numerator and the denominator of $Y$. The transformation $m$ (resp. $m^{-1}$ ) actually corresponds to both of $M$ and $\left(S_{\xi}\right)^{2} M$ (resp. $M^{-1}$ and $\left.\left(S_{\xi}\right)^{2} M^{-1}\right)$. Furthermore, whichever value $\xi$ takes, we have $M=S_{\xi}\left(S_{1} S_{\xi}\right)^{2} S_{0}\left(S_{1} S_{\xi}\right)$. Lastly, the transformation $h$ corresponds to the transformation

$$
H \quad\left\{\begin{array}{l}
(x, y) \rightarrow(u, Y)=(x,-y) \\
\left(\theta_{0}, \theta_{\infty}\right) \rightarrow\left(\bar{\theta}_{0}, \bar{\theta}_{\infty}\right)=\left(-\theta_{\infty}-2,-\theta_{\infty}\right) .
\end{array}\right.
$$

and the transformation $\left(S_{\xi}\right)^{2} H$.

The Hamiltonian expression (1.8) of $P_{\mathrm{III}^{\prime}}$ and the transformation groups $\mathbf{G}_{H}^{\prime}$, $\mathbf{G}^{\prime}$ also play important roles in the forthcoming paper [8].

\subsection{Main results}

In this paper, if we do not comment especially, "a rational function" means a single-valued algebraic function defined on a Riemann sphere $\mathbf{P}$, and "an algebraic function" means a many-valued algebraic function defined on $\mathbf{P}$.

TheOREM 1. $P_{\mathrm{III}}(\alpha, \beta, \gamma, \delta)$ has a rational solution if and only if $\alpha=\gamma=0$ or $\beta=\delta=0$.

THEOREM 2. Assume that $\gamma \delta \neq 0$.

(1) $P_{\mathrm{III}}\left(\theta_{0}, \theta_{\infty}\right)\left(\left(\theta_{0}, \theta_{\infty}\right) \in \boldsymbol{C}^{2}\right)$ does not have rational solutions.

(2) $P_{\text {III' }}\left(\theta_{0}, \theta_{\infty}\right)\left(\left(\theta_{0}, \theta_{\infty}\right) \in \boldsymbol{C}^{2}\right)$ has algebraic solutions if and only if there exists an integer $I$ such that $\theta_{\infty}-\theta_{0}-1=2 I$ or $\theta_{\infty}+\theta_{0}+1=2 I$.

(3) If $P_{\mathrm{III}}\left(\theta_{0}, \theta_{\infty}\right)$ has algebraic solutions, then the number of algebraic solutions is one or two. $P_{\mathrm{III}}\left(\theta_{0}, \theta_{\infty}\right)$ has two algebraic solutions if and only if there exist two integers $I$ and $J$ such that $\theta_{\infty}-\theta_{0}-1=2 I$ and $\theta_{\infty}+\theta_{0}+1=2 J$.

(4) Let

$$
\begin{aligned}
& D_{+}=\{(-5 / 2,1 / 2),(1 / 2,-1 / 2)\}, D_{-}=\{(-5 / 2,-1 / 2),(1 / 2,1 / 2)\}, \\
& \begin{array}{r}
\alpha_{+}=1, \alpha_{-}=i, \\
\Delta_{ \pm}=\{((L-2 K-1) / 2, \pm(L+2 K+1) / 2) \mid K \text { and } L \text { are integers such that } \\
K \geq 2, L=-2,-4, \cdots,-2 K\} \\
\cup\{((L-2 K-1) / 2, \pm(L+2 K+1) / 2) \mid K \text { and } L \text { are integers such that } \\
K \leq-2, L=0,2, \cdots, 2(-K-1)\},
\end{array}
\end{aligned}
$$

where double signs correspond with eath other. Then, algebraic solutions are classified as in the table $\mathrm{I}$. In the types $\mathrm{III}_{ \pm}, \mathrm{IV}_{ \pm}$, the values of $N, \varepsilon_{j}, b_{j}(j=1, \cdots, N)$ depend 
on the values of $\theta_{0}, \theta_{\infty}$ and $\alpha_{ \pm}$.

\begin{tabular}{|c|c|c|}
\hline Type & Conditions of $\left(\theta_{0}, \theta_{\infty}\right)$ & Forms of algebraic solutions \\
\hline$I_{ \pm}$ & $\theta_{\infty} \mp \theta_{0} \mp 1=0,\left(\theta_{0}, \theta_{\infty}\right) \in \mathbf{C}^{2}$ & $q=\alpha_{ \pm} \sqrt{t}$ \\
\hline $\mathrm{II}_{ \pm}$ & $\left(\theta_{0}, \theta_{\infty}\right) \in D_{ \pm}$ & $q=\alpha_{ \pm} \sqrt{t}+\theta_{\infty}$ \\
\hline $\mathrm{III}_{ \pm}$ & $\begin{array}{l}\theta_{\infty} \mp \theta_{0} \mp 1=2 \mathrm{I},\left(\theta_{0}, \theta_{\infty}\right) \in \mathbf{C}^{2} \\
\mathrm{I} \in \mathbf{Z}-\{0\},\left(\theta_{0}, \theta_{\infty}\right) \notin D_{ \pm} \cup \Delta_{ \pm}\end{array}$ & $\begin{array}{l}q=\sqrt{t}\left(\alpha_{ \pm}+\frac{1}{2} \sum_{j=1}^{N} \frac{\varepsilon_{j}}{\sqrt{t}-b_{j}}\right) \\
\left(\begin{array}{l}N \in \mathbf{Z}, N>0 \\
\varepsilon_{j}=1 \text { or }-1 \\
b_{j} \in \mathbf{C}-\{0\} \\
\text { if } j \neq j^{\prime}, \text { then } b_{j} \neq b_{j^{\prime}} \\
I=\sum_{j=1}^{N} \varepsilon_{j}\end{array}\right) \cdots(*)\end{array}$ \\
\hline $\mathrm{IV}_{ \pm}$ & $\left(\theta_{0}, \theta_{\infty}\right) \in \Delta_{ \pm}$ & $\begin{array}{l}q=\sqrt{t}\left(\alpha_{ \pm}+\frac{1}{2} \sum_{j=1}^{N} \frac{\varepsilon_{j}}{\sqrt{t}-b}\right)+\theta_{\infty} \\
\text { under the same conditions as in }(*)\end{array}$ \\
\hline
\end{tabular}

Table I. Algebraic solutions of $P_{\mathrm{III}^{\prime}}\left(\theta_{0}, \theta_{\infty}\right)$.

Remark 1.1. (1) Every algebraic solution is actually calculable from the algebraic solution of the type $I_{+}$by means of transformation group $\mathbf{G}^{\prime}$. (Refer to Propositions 3.4, 3.10 and 3.11.)

(2) When the values of $\theta_{0}$ and $\theta_{\infty}$ are restricted to real numbers, algebraic solutions are distributed as in the Figure 1.

As is well known $[13,4.1]$, when $\theta_{0}+\theta_{\infty}=0\left(\left(\theta_{0}, \theta_{\infty}\right) \in \mathbf{C}^{2}\right), P_{\mathrm{III}}\left(\theta_{0}, \theta_{\infty}\right)$ contains all solutions of a Riccati equation:

$$
\frac{d q}{d t}=-\frac{1}{t} q^{2}-\frac{\theta_{0}}{t} q+1
$$

By the change of variables: $q=(s / 2)(d / d s) \log u-\theta_{0} / 2(u \neq 0), t=-s^{2} / 4$, (1.9) is transformed into a Bessel equation:

$$
\frac{d^{2} u}{d s^{2}}+\frac{1}{s} \frac{d u}{d s}+\left(1-\frac{\theta_{0}^{2}}{s^{2}}\right) u=0(u \neq 0) .
$$

Let $\phi_{\sigma}(t) \quad(\sigma \in \mathbf{C})$ denote the one-parameter family of solutions of (1.9). 


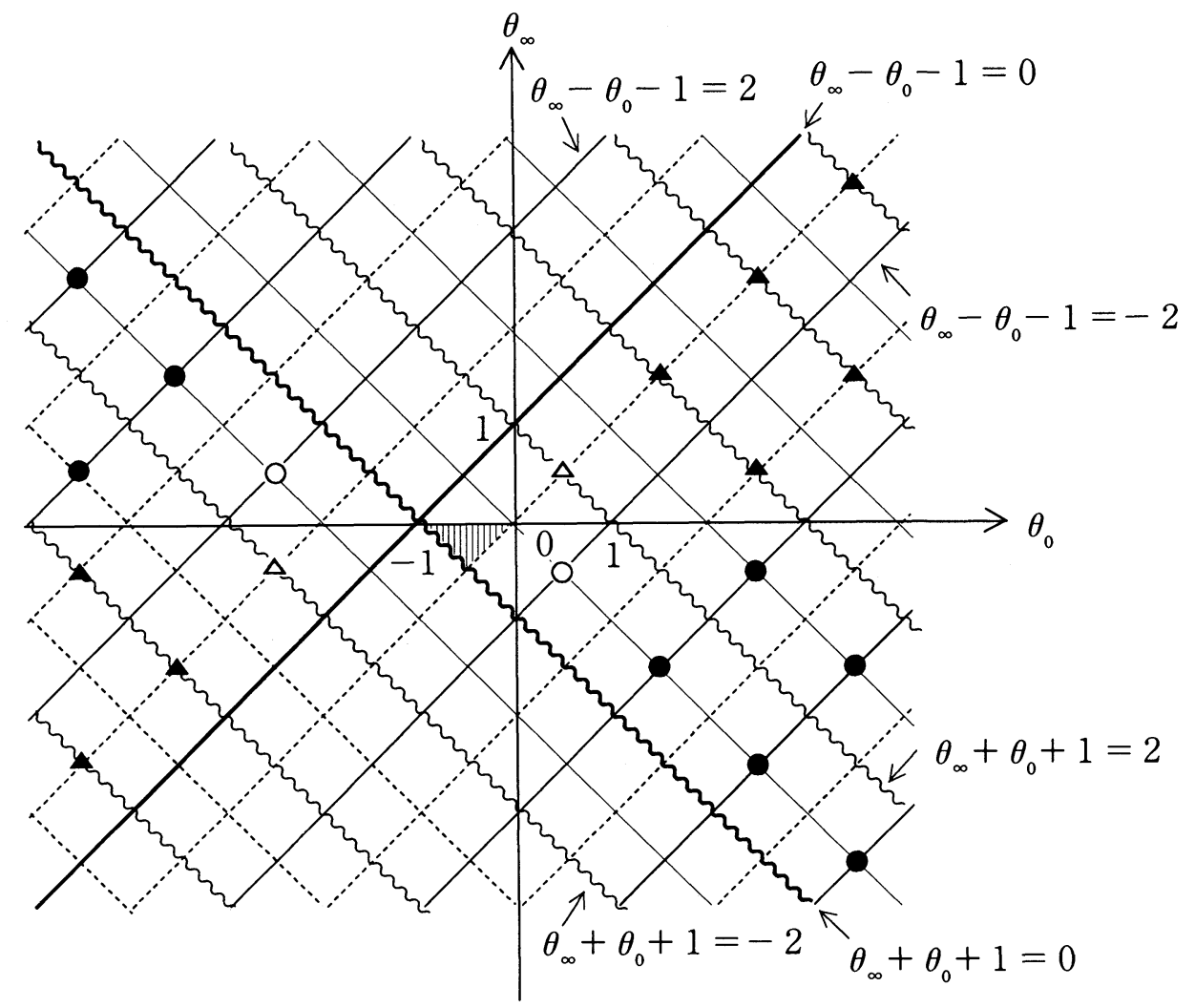

Figure 1. The distribution of algebraic solutions of $\mathrm{P}_{\mathrm{III}}\left(\theta_{0}, \theta_{\infty}\right)$ in the case $\left(\theta_{0}, \theta_{\infty}\right) \in \boldsymbol{R}^{2}$.

On the line - type $\mathrm{I}_{+}$.

On a line - type $\mathrm{III}_{+}$.

On a point $C$, type $\mathrm{II}_{+}$.

On a point $\bullet$, type $\mathrm{IV}_{+}$.
On the wavy line $\sim$, type $I_{-}$.

On a wavy line $\sim \sim$, type III_.

On a point $\Delta$, type II_. $_{\text {. }}$

On a point $\Delta$, type IV_.

याग is a fundamental cell of the Affine Weyl group of the type $B_{2}$.

TheOREM 3. Let $I$ and $J$ be any integers. If $\theta_{\infty}+\theta_{0}=2 I$ (resp. $\theta_{\infty}-\theta_{0}=2 J$ ), $P_{\mathrm{III}}\left(\theta_{0}, \theta_{\infty}\right)$ has a one-parameter family of solutions of the form

$$
\begin{gathered}
q_{I}(t)=\mathscr{Q}_{I}\left(\theta_{0}, t, \phi_{\sigma}(t)\right) \quad(\sigma \in \mathbf{C}) \\
\left(\text { resp. } \bar{q}_{J}(t)=\overline{\mathscr{Q}}_{J}\left(\theta_{0}, t, \phi_{\sigma}(-t)\right) \quad(\sigma \in \mathbf{C})\right) .
\end{gathered}
$$

Here, $\mathscr{Q}_{I}$ and $\overline{\mathscr{Q}}_{J}$ are rational functions in three variables with integer coefficients, forms of which depend on the values of $I$ and $J$ respectively. If $\theta_{\infty}+\theta_{0}=2 I$ and $\theta_{\infty}-\theta_{0}=$ $2 J$, then $P_{\mathrm{III}}\left(\theta_{0}, \theta_{\infty}\right)$ has two one-parameter families $q_{I}(t)$ and $\bar{q}_{J}(t)$ which do not have common solutions. 
Remark 1.2. (1) Theorem 3 itself does not guarantee that $q_{I}(t)$ and $\bar{q}_{J}(t)$ are all the solutions which can be expressible by solutions of Riccati equations. But, as we will show in the next paper [8], it is actually true.

(2) Okamoto $[13,4.1,4.2,4.3]$ obtained the $\tau$-function of the solutions $q_{I}(t)$ and investigated its properties, relating it to the Toda lattice equation.

In the forthcoming paper [8], by the theory of differential fields, we prove the theorem that any solution of $P_{\mathrm{III}^{\prime}}\left(\theta_{0}, \theta_{\infty}\right)$ is irreducible except for algebraic solutions of Theorem 2 and one-parameter families of solutions $q_{I}(t)$ and $\bar{q}_{J}(t)$ of Theorem 3 .

\section{Proof of Theorem 1}

First we note that the equation $P_{\mathrm{III}}(\alpha, \beta, \gamma, \delta)$ (See (1.2)) is equivalent to the equation

$$
4 t^{2} q \frac{d^{2} q}{d t^{2}}=4 t^{2}\left(\frac{d q}{d t}\right)^{2}-4 t q \frac{d q}{d t}+q^{3}(\gamma q+\alpha)+\beta t q+\delta t^{2} \quad(q \neq 0)
$$

Proof of Theorem 1. Let $q(t)$ be a rational solution of $P_{\mathrm{III}}(\alpha, \beta, \gamma, \delta)$. We suppose that $q(t)$ has a pole at $t=\infty$. Putting the Laurent expansion of $q(t)$ at $t=\infty$ into (2.1) and comparing the coefficients of the terms of the highest degree with respect to $t$, we obtain the condition $\alpha=\gamma=0$. Similarly, if $q(t)$ is holomorphic at $t=\infty$, the condition $\beta=\delta=0$ is derived. Conversely $P_{\mathrm{III}}(\alpha, 0, \gamma, 0)$ has a general solution $q=2 \lambda^{2} F /[(F-1)\{(\alpha / 4-\varepsilon) F-(\alpha / 4+\varepsilon)\}]$, where $F=\mu t^{\lambda}, \varepsilon=\sqrt{\gamma} \lambda / 2,(\lambda, \mu) \in(\mathbf{C}-\{0\}) \times \mathbf{C}$ (Okamoto [13, Proposition 1.5]). Then, if $\lambda$ is an integer, $q$ is a rational solution of $P_{\mathrm{III}}(\alpha, 0, \gamma, 0) . P_{\mathrm{III}}(0, \beta, 0, \delta)$ also has rational solutions.

\section{Proof of Theorem 2}

\subsection{Possible rational solutions of $P_{\mathrm{III}}\left(\theta_{0}, \theta_{\infty}\right)$}

From now on, we assume that $\gamma \delta \neq 0$, and we consider the canonical equation $P_{\mathrm{III}}\left(\theta_{0}, \theta_{\infty}\right)$.

Let $q(t)$ be an algebraic solution of $P_{\mathrm{III}}\left(\theta_{0}, \theta_{\infty}\right), \mathscr{R}$ the Riemann surface of $q(t), T$ a Riemann sphere $\mathbf{P}, \mathrm{pr} ; \mathscr{R} \rightarrow T$ the canonical projection. By simple calculations, we can check that any algebraic singularities of $q(t)$ on $t=b \in \mathbf{C}$ - 
$\{0\}$ is a pole. Then, $\mathscr{R}$ has branching points only on $t=0, \infty \in T$. By the Riemann-Hurwitz's formula, $\mathscr{R}$ has one branching point on each point of $t=0$ and $t=\infty$, and the two branching points have the same multiplicity. Let $n(\geq 1)$ denote this multiplicity. If we put $X=\mathbf{P}$, a Riemann sphere, and $\phi_{n}: X \rightarrow T, x \rightarrow$ $t=x^{n}$, then $\operatorname{pr}^{-1} \phi_{n}$ uniformize $\mathscr{R}$. Hence $z(x)=\left(q \phi_{n}\right)(x)$ is a rational function on $X=\mathbf{P}$. Conversely if $z(x)$ is a rational function on $X$, then $q(t)=\left(z \phi_{n}^{-1}\right)(t)$ is a $d$-sheeted algebraic function, where $d$ is a divisor of $n$.

Proposition 3.1. Let $q(t)$ be an algebraic solution of $P_{\mathrm{III}^{\prime}}\left(\theta_{0}, \theta_{\infty}\right)$. Then $q(t)$ is two-sheeted and is expanded at $t=\infty$ as

$$
q(t)=C_{1} x+C_{0}+C_{-1} x^{-1}+\cdots+C_{-k} x^{-k}+\cdots,
$$

where $x=\sqrt{t},\left(C_{1}, C_{0}\right)=\left( \pm 1,\left(\theta_{\infty}-\theta_{0}-1\right) / 4\right)$ or $\left(C_{1}, C_{0}\right)=\left( \pm i,\left(\theta_{\infty}+\theta_{0}\right.\right.$ $+1) / 4)$. For any integer $k \geq 1, C_{-k}=C_{1} C_{0} R_{k}\left(\theta_{0}, \theta_{\infty}, C_{1}, C_{0}\right)$ holds, where $R_{k}(X, Y, Z, W)$ is a polynomial in four variables with complex coefficients.

Proof. Suppose $q(t)$ is an $n$-sheeted algebraic solution with an expansion at $t=\infty$ as

$$
q(t)=C_{m} x^{m}+C_{m-1} x^{m-1}+\cdots,
$$

where $x=t^{1 / n}, m \in \mathbf{Z}, C_{m} \neq 0$. We substitute this expansion into (2.1) and compare the coefficients. If $m \leq 0$, then we cannot cancel the coefficient of $x^{2 n}$. Therefore, $m$ must be a positive integer. We also find that $m=n / 2$ and $C_{m}={ }_{4} \sqrt{1}=$ $\pm 1, \pm i$. Thus we have

(the right hand side of (2.1))

- (the left hand side of (2.1))

$$
\begin{aligned}
& =4 \sum_{4 m>p \geq 3 m}\left(\sum_{k+l+v+w=p} C_{k} C_{l} C_{v} C_{w}\right) x^{p}+4\left(-\theta_{\infty} C_{m}^{2}+\theta_{0}+1\right) C_{m} x^{3 m} \\
& +4 \sum_{3 m>p \geq 2 m}\left\{\sum_{k+l+v+w=p} C_{k} C_{l} C_{v} C_{w}-\theta_{\infty} \sum_{k+l+v=p} C_{k} C_{l} C_{v}+\left(\theta_{0}+1\right) C_{p-2 m}\right\} x^{p} \\
& +4 \sum_{2 m>p}\left\{\sum_{k+l+v+w=p} C_{k} C_{l} C_{v} C_{w}-\theta_{\infty} \sum_{k+l+v=p} C_{k} C_{l} C_{v}\right. \\
& \left.+\left(\theta_{0}+1\right) C_{p-2 m}+\frac{1}{4 m^{2}} \sum_{k+l=p}(k-2 m)(l-k) C_{k} C_{l}\right\} x^{p}=0 .
\end{aligned}
$$

Comparing the coefficients of $x^{4 m-d}(1 \leq d<m)$, we inductively obtain $C_{m-1}=\cdots=C_{1}=0$. Next, from the coefficients of $x^{3 m}$, we have $4\left(4 C_{0} C_{m}{ }^{2}-\right.$ $\left.\theta_{\infty} C_{m}{ }^{2}+\theta_{0}+1\right) C_{m}=0$. Then $C_{m}= \pm 1$ and $C_{m}= \pm i$ imply $C_{0}=\left(\theta_{\infty}-\theta_{0}\right.$ $-1) / 4$ and $C_{0}=\left(\theta_{\infty}+\theta_{0}+1\right) / 4$ respectively. Continuing this process succes- 
sively, from the coefficients of $x^{3 m-(e m+d)}(e \geq 0,1 \leq d<m)$, we obtain $C_{-e m-1}$ $=\cdots=C_{-(e+1) m+1}=0$. From the coefficients of $x^{3 m-(e+1) m}(e \geq 1)$, we find that

$$
C_{-(e+1) m}=-\left(C_{m} / 4\right) C_{0} \cdot\left(\text { a polynomial of } \theta_{0}, \theta_{\infty}, C_{m}, C_{0}, \cdots, C_{-e m}\right.
$$

with complex coefficients)

$$
=C_{m} C_{0} \cdot\left(\text { a polynomial of } \theta_{0}, \theta_{\infty}, C_{m}, C_{0}\right. \text { with complex coefficients). }
$$

By the above arguments, it follows that the expansion of $q(t)$ at $t=\infty$ must be $q(t)=\sum_{k \leq 1} C_{m k} x^{m k}=\sum_{k \leq 1} C_{m k}(\sqrt{t})^{k}$, where $x=t^{1 / 2 m}$. Hence the multiplicity $2 m$ is equal to 2 .

The arguments above and (1.3) in 1.1 lead us to the following result.

Proposition 3.2. If $q(t)$ is an algebraic solution of $P_{\mathrm{III}}\left(\theta_{0}, \theta_{\infty}\right)$, then $y(x)=$ $q\left(x^{2}\right) / x$ is a rational solution of $P_{\mathrm{III}}\left(\theta_{0}, \theta_{\infty}\right)$. Conversely, if $y(x)$ is a rational solution of $P_{\mathrm{III}}\left(\theta_{0}, \theta_{\infty}\right)$, then $q(t)=\sqrt{t} y(\sqrt{t})$ is an algebraic solution of $P_{\mathrm{III}}\left(\theta_{0}, \theta_{\infty}\right)$.

By this proposition, what we should do to find all algebraic solutions of $P_{\text {III' }}\left(\theta_{0}, \theta_{\infty}\right)$ is to find all rational solutions of $P_{\text {III }}\left(\theta_{0}, \theta_{\infty}\right)$. In Proposition 3.3, we determine all possible forms of rational solutions of $P_{\text {III }}\left(\theta_{0}, \theta_{\infty}\right)$. From Propositions $3.1,3.2$, we first obtain the following lemma.

Lemma 3.1. Let $y(x)$ be a rational solution of $P_{\mathrm{III}}\left(\theta_{0}, \theta_{\infty}\right)$. Then $y(x)$ is expanded at $x=\infty$ as

$$
y(x)=D_{0}+D_{-1} / x+\cdots+D_{-k-1} / x^{k+l}+\cdots,
$$

where $\left(D_{0}, D_{1}\right)=\left( \pm 1,\left(\theta_{\infty}-\theta_{0}-1\right) / 4\right)$ or $\left( \pm i,\left(\theta_{\infty}+\theta_{0}+1\right) / 4\right)$. For any integer $k \geq 1, D_{-k-1}=D_{0} D_{-1} \cdot R_{k}\left(\theta_{0}, \theta_{\infty}, D_{0}, D_{-1}\right)$ holds, where $R_{k}$ is the same polynomial as in Proposition 3.1 .

By simple calculation, we obtain the next lemma.

LeMma 3.2. (1) If $y(x)$ is a rational solution of $P_{\mathrm{III}}\left(\theta_{0}, \theta_{\infty}\right)$ with a pole at $x=0$, then it is expanded at $x=0$ as

$$
y(x)=\theta_{\infty} x^{-1}+E_{0}+E_{1} x+\cdots,
$$

where $\theta_{\infty} \neq 0$.

(2) If $y(x)$ is a rational solution of $P_{\mathrm{III}}\left(\theta_{0}, \theta_{\infty}\right)$ with a pole at $x=b(\in \mathbf{C}-$ 
$\{0\})$, then it is expanded at $x=b$ as

$$
y(x)=( \pm 1 / 2)(x-b)^{-1}+F_{0}+F_{1}(x-b)+\cdots .
$$

The above two lemmas give us the information on the possible forms of rational solutions of $P_{\text {III }}\left(\theta_{0}, \theta_{\infty}\right)$.

Proposition 3.3. If $y(x)$ is a rational solution of $P_{\mathrm{III}}\left(\theta_{0}, \theta_{\infty}\right)$, then it must belong to one of the eight types in the table II, where $\alpha_{+}=1$ or $-1, \alpha_{-}=i$ or $-i$, and double signs correspond with each other.

\begin{tabular}{|c|c|c|}
\hline Type & Conditions of $\left(\theta_{0}, \theta_{\infty}\right)$ & Forms of $y(x)$ 's \\
\hline $\mathrm{I}_{ \pm}$ & $\theta_{\infty} \mp \theta_{0} \mp 1=0,\left(\theta_{0}, \theta_{\infty}\right) \in \mathbf{C}^{2}$ & $y=\alpha_{ \pm}$ \\
\hline $\mathrm{II}_{ \pm}$ & $\begin{array}{c}3 \theta_{\infty} \pm \theta_{0} \pm 1=0, \theta_{\infty} \neq 0 \\
\left(\theta_{0}, \theta_{\infty}\right) \in \mathbf{C}^{2}\end{array}$ & $y=\alpha_{ \pm}+\theta_{\infty} / x$ \\
\hline $\mathrm{III}_{ \pm}$ & $\theta_{\infty} \mp \theta_{0} \mp 1=2 \mathrm{I},\left(\theta_{0}, \theta_{\infty}\right) \in \mathbf{C}^{2}$ & $\begin{array}{c}\quad y=\alpha_{ \pm}+\frac{1}{2} \sum_{j=1}^{N} \frac{\varepsilon_{j}}{x-b_{j}} \\
\left(\begin{array}{l}N \in \mathbf{Z}, N>0 \\
\varepsilon_{j}=1 \text { or }-1 \\
b_{j} \in \mathbf{C}-\{0\} \\
\text { if } j \neq j^{\prime}, \text { then } b_{j} \neq b_{j^{\prime}} \\
I=\sum_{j=1}^{N} \varepsilon_{j}\end{array}\right) \cdots(*)\end{array}$ \\
\hline $\mathrm{IV}_{ \pm}$ & $\begin{array}{c}3 \theta_{\infty} \pm \theta_{0} \pm 1=-2 I, \theta_{\infty} \neq 0 \\
\left(\theta_{0}, \theta_{\infty}\right) \in \mathbf{C}^{2}\end{array}$ & $\begin{array}{l}\qquad y=\alpha_{ \pm}+\frac{\theta_{\infty}}{x}+\frac{1}{2} \sum_{j=1}^{N} \frac{\varepsilon_{j}}{x-b_{j}} \\
\text { under the same conditions as in }(*)\end{array}$ \\
\hline
\end{tabular}

Table II. Possible types of rational solutions of $P_{\text {III }}\left(\theta_{0}, \theta_{\infty}\right)$.

Proof. By Lemma 3.1 and Lemma 3.2, the possible decompositions of $y(x)$ into partial fractions are classified into the eight types $I_{ \pm}, \cdots, I_{ \pm}$. For each type, we can derive the conditions which $\theta_{0}$ and $\theta_{\infty}$ should fulfill. Here we show the case $\mathrm{IV}_{ \pm}$only.

If $\left|b_{j}\right|<|x|$, then we have

$$
\frac{\varepsilon_{j}}{x-b_{j}}=\frac{\varepsilon_{j}}{x} \frac{1}{1-b_{j} / x}=\frac{\varepsilon_{j}}{x}\left(1+b_{j} / x+b_{j}^{2} / x^{2}+\cdots\right) .
$$


Hence, in the neighborhood of $x=\infty$, we obtain an expansion

$$
y(x)=\alpha_{ \pm}+\theta_{\infty} / x+(1 / 2)\left\{I / x+\left(\sum_{j=1}^{N} \varepsilon_{j} b_{j}\right) / x^{2}+\cdots\right\} .
$$

From Lemma 3.1, it follows that $\theta_{\infty}+I / 2=\left(\theta_{\infty} \mp \theta_{0} \mp 1\right) / 4$. Then, we have $3 \theta_{\infty} \pm \theta_{0} \pm 1=-2 I$.

Here we introduce new notations $R\left(\theta_{0}, \theta_{\infty}\right)$ and $r\left(\theta_{0}, \theta_{\infty}\right)$. Let $R\left(\theta_{0}, \theta_{\infty}\right)$ denote the set of all rational solutions of $P_{\mathrm{III}}\left(\theta_{0}, \theta_{\infty}\right)$. If $P_{\mathrm{III}}\left(\theta_{0}, \theta_{\infty}\right)$ does not have a rational solution, we consider $R\left(\theta_{0}, \theta_{\infty}\right)=\phi$. We express an element of $R\left(\theta_{0}, \theta_{\infty}\right)$ by a form $(x, y)$, where $x$ is the independent variable of $P_{\text {III }}\left(\theta_{0}, \theta_{\infty}\right)$ and $y$ is a rational solution of $P_{\text {III }}\left(\theta_{0}, \theta_{\infty}\right)$ expressed by the variable $x$. Next, let $r\left(\theta_{0}, \theta_{\infty}\right)$ denote the set of all rational solutions of $P_{\mathrm{III}}\left(\theta_{0}, \theta_{\infty}\right)$ of the types $\mathrm{I}_{+}, \cdots$, $\mathrm{IV}_{+} \cdot r\left(\theta_{0}, \theta_{\infty}\right)$ is a subset of $R\left(\theta_{0}, \theta_{\infty}\right)$. By Proposition 3.3, we must study eight types of rational solutions. But the following result decreases our labor.

Proposition 3.4. (1) The mapping

$$
\begin{gathered}
S_{i}: R\left(\theta_{0}, \theta_{\infty}\right) \rightarrow R\left(\bar{\theta}_{0}, \bar{\theta}_{\infty}\right)=R\left(\theta_{0},-\theta_{\infty}\right), \\
\quad(x, y) \rightarrow S_{i}(x, y)=(u, Y)=(i x, i y)
\end{gathered}
$$

is bijective, where $i=\sqrt{-1}$.

(2) If $(x, y)$ is a rational solution of the type $J_{ \pm}(J=I, \cdots, I V)$, then $S_{i}(x, y)$ $=(u, Y)$ is of the type $J_{\mp}$, where double signs correspond with each other.

We omit the proof. By this proposition, we may concentrate on the study of rational solutions of the types $\mathrm{I}_{+}, \cdots, \mathrm{IV}_{+}$in the following.

\subsection{Existence and uniqueness}

First, we note that $P_{\text {III }}\left(\theta_{0}, \theta_{\infty}\right)$ is equivalent to the equation

$$
x y \frac{d^{2} y}{d x^{2}}=x\left(\frac{d y}{d x}\right)^{2}-y \frac{d y}{d x}+4\left(-\theta_{\infty} y^{2}+\theta_{0}+1\right) y+4 x y^{4}-4 x(y \neq 0) \text {. }
$$

Proposition 3.5. (1) (Existence and uniqueness of the type $\mathrm{I}_{+}$) If $y(x)=1$ or $y(x)=-1$ belongs to $r\left(\theta_{0}, \theta_{\infty}\right)$, then $\left(\theta_{0}, \theta_{\infty}\right)\left(\in \mathbf{C}^{2}\right)$ satisfies the condition $\theta_{\infty}-$ $\theta_{0}-1=0$. Conversely, if $\theta_{\infty}-\theta_{0}-1=0\left(\left(\theta_{0}, \theta_{\infty}\right) \in \mathbf{C}^{2}\right)$, then $r\left(\theta_{0}, \theta_{\infty}\right)=$ $\{y(x)= \pm 1\}$.

(2) (Existence and uniqueness of the type $\mathrm{II}_{+}$) If $y(x)=1+\theta_{\infty} / x$ or $y(x)=$ 
$-1+\theta_{\infty} / x$ belongs to $r\left(\theta_{0}, \theta_{\infty}\right)$, then $\left(\theta_{0}, \theta_{\infty}\right)=(-5 / 2,1 / 2)$ or $(1 / 2,-1 / 2)$. Conversely, if $\left(\theta_{0}, \theta_{\infty}\right)=(-5 / 2,1 / 2)$ or $(1 / 2,-1 / 2)$, then $r\left(\theta_{0}, \theta_{\infty}\right)=$ $\left\{y(x)= \pm 1+\theta_{\infty} / x\right\}$.

Proof. We prove (2) only. The proof of (1) is the same as that of (2). If $P_{\text {III }}\left(\theta_{0}, \theta_{\infty}\right)$ has $y(x)=1+\theta_{\infty} / x$ or $y(x)=-1+\theta_{\infty} / x$ as a solution, it follows from Proposition 3.3 that $3 \theta_{\infty}+\theta_{0}+1=0$ and $\theta_{\infty} \neq 0$. On the other hand, substituting $y(x)$ into (3.1) and comparing the coefficients of $x^{-2}$, we obtain $4 \theta_{\infty}{ }^{2}=1$. Therefore $\left(\theta_{0}, \theta_{\infty}\right)=(-5 / 2,1 / 2)$ or $(1 / 2,-1 / 2)$. Conversely, if $\left(\theta_{0}, \theta_{\infty}\right)=(-5 / 2,1 / 2)$ or $(1 / 2,-1 / 2)$, then we have $3 \theta_{\infty}+\theta_{0}+1=0$ and $4 \theta_{\infty}{ }^{2}=1$. At this time, $y(x)= \pm 1+\theta_{\infty} / x$ satisfy (3.1). Moreover, Lemma 3.1 ensure that $r\left(\theta_{0}, \theta_{\infty}\right)$ does not contain rational solutions of other types.

Proposition 3.6 (Existence and uniqueness of the type $\mathrm{III}_{+}$and the type $\mathrm{IV}_{+}$ (1)). (1) If $P_{\mathrm{III}}\left(\theta_{0}, \theta_{\infty}\right)$ has a rational solution of the type $\mathrm{III}_{+}$, then it most be that $r\left(\theta_{0}, \theta_{\infty}\right)=\left\{y(x)= \pm 1+(1 / 2) \sum_{j=1}^{N} \varepsilon_{j} /\left(x \mp b_{j}\right)\right\}$, where double signs correspond with each other.

(2) If $P_{\mathrm{III}}\left(\theta_{0}, \theta_{\infty}\right)$ has a rational solution of the type $\mathrm{IV}_{+}$, than it must be that $r\left(\theta_{0}, \theta_{\infty}\right)=\left\{y(x)=\mp 1+\theta_{\infty} / x+(1 / 2) \sum_{j=1}^{N} \varepsilon_{j} /\left(x \mp b_{j}\right)\right\}$, where double signs correspond with each other.

Proof. We prove (2) only. Since the transformation $\left(S_{i}\right)^{2}: R\left(\theta_{0}, \theta_{\infty}\right) \rightarrow$ $R\left(\theta_{0}, \theta_{\infty}\right),(x, y) \rightarrow(u, Y)=(-x,-y)$ is bijective, if $y(x)=1+\theta_{\infty} / x+$ $(1 / 2) \sum_{j=1}^{N} \varepsilon_{j} /\left(x-b_{j}\right)$ is a solution of $P_{I I I}\left(\theta_{0}, \theta_{\infty}\right)$, then $Y(u)=-1+\theta_{\infty} / u+$ $(1 / 2) \sum_{j=1}^{N} \varepsilon_{j} /\left(u+b_{j}\right)$ is also a solution of $P_{\mathrm{III}}\left(\theta_{0}, \theta_{\infty}\right)$, and vice versa. Next, Lemma 3.1 ensure that for a fixed $\left(\theta_{0}, \theta_{\infty}\right)$ a rational solution expressed as $y=1$ $+D_{-1} / x+D_{-2} / x^{2}+\cdots$ or $y=-1+D_{-1} / x+D_{-2} / x^{2}+\cdots$ is uniquely determined.

Remark 3.1. From Propositions 3.4, 3.5 and 3.6, we see that $R\left(\theta_{0}, \theta_{\infty}\right)$ has only the following four possibilities:

$$
\begin{array}{r}
R\left(\theta_{0}, \theta_{\infty}\right)=\phi . \\
\left.R\left(\theta_{0}, \theta_{\infty}\right)=\text { two rational solutions of the type } J_{+}\right\} \\
\left(J_{+}=\mathrm{I}_{+}, \cdots, \mathrm{IV}_{+}\right) . \\
\left.R\left(\theta_{0}, \theta_{\infty}\right)=\text { two rational solutions of the type } K_{-}\right\} \\
\left(K_{-}=\mathrm{I}_{-}, \cdots, \mathrm{IV}_{-}\right) .
\end{array}
$$


(iv)

$$
\begin{aligned}
R\left(\theta_{0}, \theta_{\infty}\right)= & \left\{\text { two rational solutions of the type } J_{+}\right\} \\
\cup & \left\{\text { two rational solutions of type } K_{-}\right\} \\
& \left(J_{+}=\mathrm{I}_{+}, \cdots, \mathrm{IV}_{+}, K_{-}=\mathrm{I}_{-}, \cdots, \mathrm{IV}_{-}\right) .
\end{aligned}
$$

From now on, we study the transformations $S_{0}, H$ and $M$ in order to determine necessary and sufficient conditions for rational solutions of the types $\mathrm{III}_{+}$, $\mathrm{IV}_{+}$to exist. The next lemma is prepared for the proofs of Proposition 3.7 and Proposition 3.9. We omit the proof of it.

Lemma 3.3. Let $y(x)= \pm 1+P(x) / Q(x)$ be a rational solution of the type $J_{+}\left(J_{+}=\mathrm{II}_{+}, \cdots, \mathrm{IV}_{+}\right)$. Here $P(x)$ and $Q(x)$ are polynomials with complex coefficients. We do not assume that $P(x)$ and $Q(x)$ are coprime.

(1) If $Q(0) \neq 0$, then $y(x)$ is of the type III $_{+}$.

(2) If $Q(0)=0$ and $P(0) \neq 0$, then $y(x)$ is of the type $\mathrm{II}_{+}$or the type $\mathrm{IV}_{+}$.

Proposition 3.7. (1) The mapping

$$
\begin{array}{r}
S_{0}: r\left(\theta_{0}, \theta_{\infty}\right) \rightarrow r\left(\bar{\theta}_{0}, \bar{\theta}_{\infty}\right)=r\left(-\theta_{\infty}-1,-\theta_{0}-1\right), \\
(x, y) \rightarrow S_{0}(x, y)=(x, Y)=(x,-1 / y)
\end{array}
$$

is bijective.

(2) Let $y$ be a type $\mathrm{IV}_{+}$rational solution of $P_{\mathrm{III}}\left(\theta_{0}, \theta_{\infty}\right)$, where $3 \theta_{\infty}+\theta_{0}+1=$ $-2 I(I \in \mathbf{Z}), \theta_{\infty} \neq 0$, and let $S_{0}(x, y)=(x, Y)$. Then $Y$ is a type $\mathrm{III}_{+}$rational solution of $P_{\mathrm{III}}\left(\bar{\theta}_{0}, \bar{\theta}_{\infty}\right)$ with the condition $Y(0)=0$, where $\bar{\theta}_{\infty}-\bar{\theta}_{0}-1=2 \bar{I}(\bar{I} \in$ $\mathbf{Z}-\{0\})$.

Proof. (1) It is obvious.

(2) Substituting $y= \pm 1+\theta_{\infty} / x+(1 / 2) \sum_{j=1}^{N} \varepsilon_{j} /\left(x \mp b_{j}\right)$ into $Y=-1 / y$ $=\mp 1+( \pm y-1) / y$, we obtain $Y(x)=\mp 1+P(x) / Q(x)$, where

$$
\begin{aligned}
& P(x)=( \pm y-1) x \Pi_{j=1}^{N}\left(x \mp b_{j}\right) \in \mathbf{C}[x] \\
& Q(x)=y x \Pi_{j=1}^{N}\left(x \mp b_{j}\right) \in \mathbf{C}[x] \\
& \operatorname{deg} P \leq N, \operatorname{deg} Q=N+1 .
\end{aligned}
$$

Since $Q(0)=\theta_{\infty} \Pi_{j=1}^{N}\left(\mp b_{j}\right) \neq 0$, by Lemma $3.3, Y(x)$ is of the type $\mathrm{III}_{+}$. Then, we have $\bar{\theta}_{\infty}-\bar{\theta}_{0}-1=2 \bar{I}$, where $\bar{I} \in \mathbf{Z}-\{0\}$ because of Proposition 3.5 (1). Next, if we assume $Y(0) \neq 0$, from $S_{0}(x, Y)=(x, y)$ and the similar argument as the above, we obtain that $y$ is of the type III $_{+}$. This is a contradiction. Then $Y(0)=0$ must hold. 
COROLlary 3.1. We consider a set

$$
\tilde{D}=\left\{((L-2 K-1) / 2,(L+2 K+1) / 2) \in \mathbf{R}^{2} \mid K, L \in \mathbf{Z}, K \neq 0\right\} .
$$

If $P_{\mathrm{III}}\left(\theta_{0}, \theta_{\infty}\right)$ has a rational solution of the type $\mathrm{IV}_{+}$, then $\left(\theta_{0}, \theta_{\infty}\right) \in \tilde{D}$.

Proof. By Proposition $3.7(2)$, if $(x, y)$ is a type $\mathrm{IV}_{+}$rational solution of $P_{\mathrm{III}}\left(\theta_{0}, \theta_{\infty}\right)$, then $S_{0}(x, y)=(x, Y)$ is a type $\mathrm{III}_{+}$rational solution of $P_{\mathrm{III}}\left(\bar{\theta}_{0}, \bar{\theta}_{\infty}\right)$, and $\left(\bar{\theta}_{0}, \bar{\theta}_{\infty}\right)$ satisfies a relation $\bar{\theta}_{\infty}-\bar{\theta}_{0}-1=2 \bar{I}(\bar{I} \in \mathbf{Z}-\{0\})$. Hence,

$$
\theta_{\infty}-\theta_{0}-1=\left(-\bar{\theta}_{0}-1\right)-\left(-\bar{\theta}_{\infty}-1\right)-1=\bar{\theta}_{\infty}-\bar{\theta}_{0}-1=2 \bar{I} .
$$

On the other hand, we have $3 \theta_{\infty}+\theta_{0}+1=-2 I(I \in \mathbf{Z})$. By the both equalities, we obtain $\theta_{0}=(-I-3 \bar{I}-2) / 2, \theta_{\infty}=(-I+\bar{I}) / 2$. Putting $-I-\bar{I}-1$ $=L$ and $\bar{I}=K(\neq 0)$, we obtain $\theta_{0}=(L-2 K-1) / 2, \theta_{\infty}=(L+2 K+1) / 2$.

Remark 3.2. A point $((L-2 K-1) / 2,(L+2 K+1) / 2)$ is an intersection of two lines $\theta_{\infty}-\theta_{0}-1=2 K$ and $\theta_{\infty}+\theta_{0}=L$ in the real plane $\mathbf{R}^{2}$.

From the table II of Proposition 3.3, Proposition 3.5 (2) and the Corollary 3.1 , we obtain

Corollary 3.2. Let $\Sigma_{ \pm}=\left\{\left(\theta_{0}, \theta_{\infty}\right) \in \mathbf{C}^{2} \mid\right.$ there exists an integer I such that $\left.\pm I \geq 0, \theta_{\infty}-\theta_{0}-1=2 I\right\}$. If $r\left(\theta_{0}, \theta_{\infty}\right)=\phi$, then $\left(\theta_{0}, \theta_{\infty}\right) \in \Sigma_{+} \cup \Sigma_{-}$.

Proposition 3.8. (1) The mapping

$$
\begin{aligned}
H: r\left(\theta_{0}, \theta_{\infty}\right) & \rightarrow r\left(\bar{\theta}_{0}, \bar{\theta}_{\infty}\right)=r\left(-\theta_{0}-2,-\theta_{\infty}\right), \\
(x, y) & \rightarrow H(x, y)=(x, Y)=(x,-y)
\end{aligned}
$$

is bijective.

(2) If $(x, y)$ is a rational solution of the type $J_{+}\left(J_{+}=\mathrm{I}_{+}, \cdots, \mathrm{IV}_{+}\right)$, then $H(x, y)$ $=(x, Y)$ is also a rational solution of the type $J_{+}$.

We omit the proof of this proposition. This proposition implies that the set $r\left(\theta_{0}, \theta_{\infty}\right) \quad\left(\left(\theta_{0}, \theta_{\infty}\right) \in \Sigma_{+}\right)$are uniquely determined by the set $r\left(\theta_{0}, \theta_{\infty}\right) \quad\left(\left(\theta_{0}, \theta_{\infty}\right)\right.$ $\left.\in \Sigma_{-}\right)$, because $\bar{\theta}_{\infty}-\bar{\theta}_{0}-1=\left(-\theta_{\infty}\right)-\left(-\theta_{0}-2\right)-1=-\left(\theta_{\infty}-\theta_{0}-1\right)$. Therefore, we may investigate the set $r\left(\theta_{0}, \theta_{\infty}\right)$ only in the case $\left(\theta_{0}, \theta_{\infty}\right) \in \Sigma_{\text {. }}$. 
Proposition 3.9. (1) The mapping

$$
\begin{gathered}
M: r\left(\theta_{0}, \theta_{\infty}\right) \rightarrow r\left(\bar{\theta}_{0}, \bar{\theta}_{\infty}\right)=r\left(\theta_{0}+1, \theta_{\infty}-1\right), \\
(x, y) \rightarrow M(x, y)=(x, Y) \\
=\left(x, \frac{1}{y} \frac{(x / 2)(d y / d x)+x y^{2}-\left(\theta_{0}+3 / 2\right) y+x}{(x / 2)(d y / d x)+x y^{2}-\left(\theta_{\infty}-1 / 2\right) y+x}\right)
\end{gathered}
$$

is bijective.

(2) If $(x, y)$ is a rational solution of the type $J_{+}\left(J_{+}=\mathrm{I}_{+}, \cdots, \mathrm{IV}_{+}\right)$, then $M(x, y)$ $=(x, Y)$ is a rational solution as in the table III. Here double signs correspond with each other, and $\left(\theta_{0}, \theta_{\infty}\right) \in \Sigma_{-}$.

Proof. (1) By Lemma 3.1, any rational solution $y(x)$ of the type $J_{+}\left(J_{+}=\right.$ $\left.\mathrm{I}_{+}, \cdots, \mathrm{IV}_{+}\right)$of $P_{\mathrm{III}}\left(\theta_{0}, \theta_{\infty}\right)$ is developed as

$$
y(x)= \pm 1+D_{-1} / x+\cdots+D_{-k-1} / x^{k+1}+\cdots
$$

at $x=\infty$. Then it follows that

$$
(x / 2)(d y / d x)+x y^{2}-A y+x=2 x+O(1) \neq 0,
$$

at $x=\infty$, whichever $A=\theta_{0}+3 / 2$ or $A=\theta_{\infty}-1 / 2$. Therefore we can apply $M$ to any solution in $r\left(\theta_{0}, \theta_{\infty}\right)$. By a similar reason, the inverse transformation $M^{-1}$ is applicable to any solution in $r\left(\bar{\theta}_{0}, \bar{\theta}_{\infty}\right)$. Therefore $M$ is bijective.

(2) We prove only the case $J_{+}=$III $_{+}$. Proofs of the other cases are done in the same ways. First, by the assumption $\left(\theta_{0}, \theta_{\infty}\right) \in \Sigma_{-}$and Proposition 3.5 (1), we have $\theta_{\infty}-\theta_{0}-1=2 \mathrm{I} \leq-2$, and $\bar{\theta}_{\infty}-\bar{\theta}_{0}-1=\left(\theta_{\infty}-1\right)-\left(\theta_{0}+1\right)-1$ $=2(\mathrm{I}-1) \leq-4$. Therefore, from Proposition 3.5, $Y$ must be of the type $\mathrm{III}_{+}$or the type $\mathrm{IV}_{+}$. Next, we substitute $y= \pm 1+(1 / 2) \sum_{j=1}^{N} \varepsilon_{j} /\left(x \mp b_{j}\right)$ into

$$
\begin{aligned}
Y & =\frac{1}{y} \frac{(x / 2)(d y / d x)+x y^{2}-\left(\theta_{0}+3 / 2\right) y+x}{(x / 2)(d y / d x)+x y^{2}-\left(\theta_{\infty}-1 / 2\right) y+x} \\
& =\frac{1}{y}+\frac{\theta_{\infty}-\theta_{0}-2}{(x / 2)(d y / d x)+x y^{2}-\left(\theta_{\infty}-1 / 2\right) y+x} .
\end{aligned}
$$

Here we note that

$$
\frac{1}{y}= \pm 1+\frac{\mp y+1}{y}= \pm 1+\frac{P_{1}(x)}{Q_{1}(x)}
$$

where

$$
P_{1}(x)=(\mp y+1) \Pi_{j=1}^{N}\left(x \mp b_{j}\right) \in \mathbf{C}[x]
$$




$$
\begin{aligned}
& Q_{1}(x)=y \Pi_{j=1}^{N}\left(x \mp b_{j}\right) \in \mathbf{C}[x] \\
& \operatorname{deg} P_{1}=N-1, \quad \operatorname{deg} Q_{1}=N .
\end{aligned}
$$

\begin{tabular}{|c|c|c|}
\hline $\begin{array}{l}\text { Type of } \\
y(x)\end{array}$ & $\begin{array}{c}\text { Conditions of }\left(\theta_{0}, \theta_{\infty}\right) \in \Sigma_{-} \\
\text {Form of } y(x)\end{array}$ & $\begin{array}{c}\text { Conditions of }\left(\bar{\theta}_{0}, \bar{\theta}_{\infty}\right) \in \Sigma_{-} \\
\text {Type of } Y(x)\end{array}$ \\
\hline $\mathrm{I}_{+}$ & $\begin{array}{c}\theta_{\infty}-\theta_{0}-1=0 \\
y= \pm 1\end{array}$ & $\begin{array}{c}\bar{\theta}_{\infty}-\bar{\theta}_{0}-1=-2 \\
\text { (i) If }\left(\bar{\theta}_{0}, \bar{\theta}_{\infty}\right) \neq(1 / 2,-1 / 2) \text {, then } \\
Y \text { is of the type } \mathrm{III}_{+} \cdot \\
\text { Here } Y(0)=0 \text { if and only if } \\
\left(\bar{\theta}_{0}, \bar{\theta}_{\infty}\right)=(-1 / 2,-3 / 2) \text {. } \\
\text { (ii) If }\left(\bar{\theta}_{0}, \bar{\theta}_{\infty}\right)=(1 / 2,-1 / 2) \text {, then } \\
Y \text { is of the type } \mathrm{II}_{+} .\end{array}$ \\
\hline $\mathrm{II}_{+}$ & $\begin{array}{c}\left(\theta_{0}, \theta_{\infty}\right)=(1 / 2,-1 / 2) \\
y= \pm 1+(-1 / 2) / x\end{array}$ & $\begin{array}{c}\left(\bar{\theta}_{0}, \bar{\theta}_{\infty}\right)=(3 / 2,-3 / 2) \\
Y \text { is of the type } \mathrm{IV}_{+}\end{array}$ \\
\hline $\mathrm{III}_{+}$ & $\begin{array}{c}\theta_{\infty}-\theta_{0}-1=2 I \\
\left(I=\sum_{j=1}^{N} \varepsilon_{j}<0\right) \\
y= \pm 1+(1 / 2) \sum_{j=1}^{N} \varepsilon_{j} /\left(x \mp b_{j}\right)\end{array}$ & $\begin{array}{l}\bar{\theta}_{\infty}-\bar{\theta}_{0}-1=2(I-1) \\
\text { (i) If } \theta_{0} \neq 1 / 2 \text { and } y(0) \neq 0 \text {, then } \\
Y \text { is of the type } \mathrm{III}_{+} \cdot \\
Y(0)=0 \text { if and only if } \theta_{0}=-3 / 2 . \\
\text { (ii) If } \theta_{\infty}=1 / 2 \text { and } y(0) \neq 0 \text {, then } \\
Y \text { is of the type } \mathrm{IV}_{+} \cdot \\
\text { (iii) If } y(0)=0 \text {, then } Y \text { is of } \\
\text { the type } \mathrm{III}_{+} \text {or the type } \mathrm{IV}_{+} \cdot\end{array}$ \\
\hline $\mathrm{IV}_{+}$ & $\begin{array}{c}\left(\theta_{0}, \theta_{\infty}\right)=((L-2 K-1) / 2, \\
(L+2 K+1) / 2) \in \tilde{D} \\
K<0 \\
y=z+\theta_{\infty} / x\left(\theta_{\infty} \neq 0\right), \\
\text { where } \\
z= \pm 1+(1 / 2) \sum_{j=1}^{N} \varepsilon_{j} /\left(x \mp b_{j}\right)\end{array}$ & $\begin{array}{c}\left(\bar{\theta}_{0}, \bar{\theta}_{\infty}\right)=((\bar{L}-2 \bar{K}-1) / 2, \\
(\bar{L}+2 \bar{K}+1) / 2) \in \tilde{D} \\
\bar{K}=K-1<0, \bar{L}=L \\
\text { (i) If } \theta_{\infty} \neq-1 / 2 \text { and } z(0) \neq 0 \text {, then } \\
Y \text { is of the type } \mathrm{III}_{+} \cdot \\
\text { (ii) If } \theta_{\infty}=-1 / 2 \text { or } z(0)=0 \text {, then } \\
Y \text { is of the type } \mathrm{IV}_{+} \cdot\end{array}$ \\
\hline
\end{tabular}

Table III. Actions of the mapping $M$.

Particularly, we have

$$
P_{1}(0)=(\mp y(0)+1) \Pi_{j=1}^{N}\left(\mp b_{j}\right), Q_{1}(0)=y(0) \Pi_{j=1}^{N}\left(\mp b_{j}\right) .
$$

Furthermore, we note that 


$$
\begin{aligned}
& \frac{\theta_{\infty}-\theta_{0}-2}{(x / 2)(d y / d x)+x y^{2}-\left(\theta_{\infty}-1 / 2\right) y+x} \\
& \quad=\frac{\theta_{\infty}-\theta_{0}-2}{x\left\{(1 / 2)(d y / d x)+y^{2}+1\right\}-\left(\theta_{\infty}-1 / 2\right) y}=\frac{P_{2}(x)}{Q_{2}(x)},
\end{aligned}
$$

where

$$
\begin{aligned}
P_{2}(x)= & \left(\theta_{\infty}-\theta_{0}-2\right) \Pi_{j=1}^{N}\left(x \mp b_{j}\right)^{2} \in \mathbf{C}[x] \\
Q_{2}(x)= & x\left\{(1 / 2)(d y / d x)+y^{2}+1\right\} \Pi_{j=1}^{N}\left(x \mp b_{j}\right)^{2} \\
& \quad-\left(\theta_{\infty}-1 / 2\right) y \Pi_{j=1}^{N}\left(x \mp b_{j}\right)^{2} \in \mathbf{C}[x]
\end{aligned}
$$

$$
\operatorname{deg} P_{2}=2 N, \quad \operatorname{deg} Q_{2}=2 N+1 .
$$

In particular, we obtain

$$
\begin{aligned}
& P_{2}(0)=\left(\theta_{\infty}-\theta_{0}-2\right) \Pi_{j=1}^{N}\left(\mp b_{j}\right)^{2}=(2 I-1) \Pi_{j=1}^{N} b_{j}{ }^{2} \neq 0 \\
& Q_{2}(0)=-\left(\theta_{\infty}-1 / 2\right) y(0) \Pi_{j=1}^{N} b_{j}{ }^{2} .
\end{aligned}
$$

Since $Y= \pm 1+P_{1}(x) / Q_{1}(x)+P_{2}(x) / Q_{2}(x)$, it follows from (3.3), (3,4) and Lemma 3.3 that

(i) When $\theta_{\infty} \neq 1 / 2$ and $y(0) \neq 0, Y$ is of the type $\mathrm{III}_{+}$.

(ii) When $\theta_{\infty}=1 / 2$ and $y(0) \neq 0, Y$ is of the type $\mathrm{IV}_{+}$.

(iii) When $y(0)=0, Y$ is either of the type $\mathrm{III}_{+}$or of the type $\mathrm{IV}_{+}$.

In the case (i), (3.2) imply that

$$
Y(0)=\frac{1}{y(0)} \frac{-\left(\theta_{0}+3 / 2\right) y(0)}{-\left(\theta_{\infty}-1 / 2\right) y(0)}=\frac{\left(\theta_{0}+3 / 2\right)}{\left(\theta_{\infty}-1 / 2\right)} \frac{1}{y(0)}=0
$$

if and only if $\theta_{0}=-3 / 2$.

Here we prepare a simple lemma for Proposition 3.10 .

Lemma 3.4. Let $y=z+\theta_{\infty} / x\left(\theta_{\infty} \neq 0\right)$ be a rational solution of the type $\mathrm{IV}_{+}$ of $P_{\mathrm{III}}\left(\theta_{0}, \theta_{\infty}\right)$, where $z= \pm 1+(1 / 2) \sum_{j=1}^{N} \varepsilon_{j} /\left(x \mp b_{j}\right)$. If $\theta_{\infty} \neq \pm 1 / 2$, then we have $z(0)=0$.

Proof. The solution $y(x)$ is developed at $x=0$ as $y=\theta_{\infty} / x+z(0)+O(x)$. We substitute this expansion into the equation (3.1). Comparing the coefficients of $x^{-2}$, we obtain $\left(4 \theta_{\infty}{ }^{2}-1\right) z(0)=0$. Therefore, if $\theta_{\infty} \neq \pm 1 / 2$, then $z(0)=0$ must hold. 
From Propositions $3.5, \cdots, 3.9$ and Lemma 3.4, we can derive the following final result about the existence of rational solutions of the type $\mathrm{III}_{+}$and the type IV $V_{+}$

Proposition 3.10 (Existence and uniqueness of the type $\mathrm{III}_{+}$and the type $\left.\mathrm{IV}_{+}(2)\right)$. We set

$$
\begin{gathered}
E_{+}=\{((L-2 K-1) / 2,(L+2 K+1) / 2) \mid K \text { and } L \text { are integers } \\
\text { such that } K \geq 2, L=-2,-4, \cdots,-2 K\} \\
E_{-}=\{((L-2 K-1) / 2,(L+2 K+1) / 2) \mid K \text { and } L \text { are integers } \\
\text { such that } K \leq-2, L=0,2, \cdots, 2(-K-1)\} \\
E=E_{+} \cup E_{-} \cup\{(-5 / 2,1 / 2),(1 / 2,-1 / 2)\} \\
\Sigma_{*}=\left\{\left(\theta_{0}, \theta_{\infty}\right) \in \mathbf{C}^{2} \mid \text { there exists a nonzero integer } I\right. \\
\text { such that } \left.\theta_{\infty}-\theta_{0}-1=2 I\right\} .
\end{gathered}
$$

(1) If $r\left(\theta_{0}, \theta_{\infty}\right)$ contains a rational solution of the type $\mathrm{III}_{+}$, then it must be that $\left(\theta_{0}\right.$, $\left.\theta_{\infty}\right) \in \Sigma_{*}-E$. Conversely, if $\left(\theta_{0}, \theta_{\infty}\right) \in \Sigma_{*}-E$, then $r\left(\theta_{0}, \theta_{\infty}\right)=\{$ two rational solutions of the type $\left.\mathrm{III}_{+}\right\}$.

(2) If $r\left(\theta_{0}, \theta_{\infty}\right)$ contains a rational solution of the type $\mathrm{IV}_{+}$, then it must be that $\left(\theta_{0}\right.$, $\left.\theta_{\infty}\right) \in E_{+} \cup E_{-}$. Conversely, if $\left(\theta_{0}, \theta_{\infty}\right) \in E_{+} \cup E_{-}$, then $r\left(\theta_{0}, \theta_{\infty}\right)=\{$ two rational solutions of the type $\left.\mathrm{IV}_{+}\right\}$.

Proof. We prove (1) and (2) simultaneously. Refer to the figure 1 in the Remark 1.1 after Theorem 2. By Propositon 3.5 and Corollary 3.2, we first note that if $r\left(\theta_{0}, \theta_{\infty}\right)$ contains rational solutions of the type $\mathrm{III}_{+}$or the type $\mathrm{IV}_{+}$, then $\left(\theta_{0}\right.$, $\left.\theta_{\infty}\right) \in \Sigma_{*}$.

Step 1. For any integer $I$, we define a set

$$
\Pi_{I}=\left\{\left(\theta_{0}, \theta_{\infty}\right) \in \mathbf{C}^{2} \mid \theta_{\infty}-\theta_{0}-1=2 I\right\} .
$$

By their definitions, $\Sigma_{*} \cup \Pi_{0}=\Sigma_{+} \cup \Sigma_{-}, E_{+} \cup\{(-5 / 2,1 / 2)\} \subset \Sigma_{+}, E_{-}$ $\cup\{(1 / 2,-1 / 2)\} \subset \Sigma_{-}$hold. Let $P_{K, L}$ denote a point $((L-2 K-1) / 2$, $(L+$ $2 K+1) / 2$ ) in $\mathbf{C}^{2}$, where $K$ and $L$ are integers. As we mentioned in the Remark 3.2 after Corollary 3.1, $P_{K, L}$ is an intersection of a plane $\Pi_{K}$ and a plane $\theta_{\infty}+\theta_{0}$ $=L$. When $\left(\theta_{0}, \theta_{\infty}\right)=P_{K, L}$, we express $r\left(\theta_{0}, \theta_{\infty}\right)$ by $r\left(P_{K, L}\right)$.

Step 2. By Proposition 3.5, Proposition 3.9 (2) $\mathrm{I}_{+}$and Proposition 3.6 (1), it turns out that when $\left(\theta_{0}, \theta_{\infty}\right) \in \Pi_{-1}-\left\{P_{-1,-2}, P_{-1,0}\right\}, r\left(\theta_{0}, \theta_{\infty}\right)=\{$ two rational 
solutions of the type $\mathrm{III}_{+}$, which satisfy $\left.y(0) \neq 0\right\}$, and that $r\left(P_{-1,-2}\right)=$ two rational solutions of the type $\mathrm{III}_{+}$, which satisfy $\left.y(0)=0\right\}, r\left(P_{-1,0}\right)=\{$ two rational solutions of the type $\left.\mathrm{II}_{+}\right\}$.

Step 3. From now, we prove the following facts (F1), (F2), (F3) by induction. Here we assume $-K=k \geq 2$.

(F1) $r\left(\theta_{0}, \theta_{\infty}\right)=$ two rational solutions of the type $\mathrm{III}_{+}$, which satisfy $y(0) \neq$ $0\}$ for any $\left(\theta_{0}, \theta_{\infty}\right) \in \Pi_{-k}-\left\{P_{-k, L} \mid L=-2 k,-2(k-1), \cdots, 2(k-1)\right\}$

(F2) $r\left(P_{-k, L}\right)=$ two rational solutions of the type $\mathrm{III}_{+}$, which satisfy $y(0)=$ $0\}$ for $L=-2 k,-2(k-1), \cdots,-2$.

(F3) $\quad r\left(P_{-k, L}\right)=\left\{\right.$ two rational solutions of the type $\left.\mathrm{IV}_{+}\right\}$for $L=0,2, \cdots, 2(k-1)$. By proposition $3.9(1)$, we note that $M\left(r\left(\theta_{0}, \theta_{\infty}\right)\right)=r\left(\bar{\theta}_{0}, \bar{\theta}_{\infty}\right)$, where $\bar{\theta}_{\infty}-\bar{\theta}_{0}-$ $1=\left(\theta_{\infty}-\theta_{0}-1\right)-2$, and that $M\left(r\left(P_{-k, L}\right)\right)=r\left(P_{-(k+1), L}\right)$.

(i) Let $k=2$. From the results in step 2 and Proposition 3.9 (2) $\mathrm{II}_{+}, \mathrm{III}_{+}$(ii), we see that if $L=0$ or 2 , then $r\left(P_{-2, L}\right)=$ two rational solutions of the type $\left.\mathrm{IV}_{+}\right\}$. By Proposition 3.7 (2), we see that if $L=-4$ or -2 , then $r\left(P_{-2, L}\right)=$ two rational solutions of the type $\mathrm{III}_{+}$, which satisfy $y(0)=0$ \}. Hence, Proposition 3.9 (2) $\mathrm{III}_{+}$(i) tells us that for any $\left(\theta_{0}, \theta_{\infty}\right) \in \Pi_{-2}-\left\{P_{-2, L} \mid L=-4,-2,0,2\right\}$, $r\left(\theta_{0}, \theta_{\infty}\right)=\left\{\right.$ two rational solutions of the type $\mathrm{III}_{+}$, which satisfy $y(0) \neq 0$ \} holds.

(ii) We suppose that $k \geq 2$ and that (F1), (F2), (F3) hold for $k$. By Proposition 3.9 (2) $\mathrm{III}_{+}$(ii), $\mathrm{IV}_{+}$(ii) and Lemma 3.4 , we see that if $L=0,2, \cdots, 2 k$, then $r\left(P_{-(k+1), L}\right)=\left\{\right.$ two rational solutions of the type $\left.\mathrm{IV}_{+}\right\}$. By Proposition 3.7 (2), we see that if $L=-2(k+1),-2 k, \cdots,-2$, then $r\left(P_{-(k+1), L}\right)=$ two rational solutions of the type $\mathrm{III}_{+}$, which satisfy $y(0)=0$. Therefore, by Proposition 3.9 (2) $\mathrm{III}_{+}$(i), we find that if $\left(\theta_{0}, \theta_{\infty}\right) \in \Pi_{-(k+1)}-\left\{P_{-(k+1), L} \mid L=-2(k+1)\right.$, $-2 k, \cdots, 2 k\}, r\left(\theta_{0}, \theta_{\infty}\right)=$ two rational solutions of the type III $_{+}$, which satisfy $y(0) \neq 0\}$ holds.

(iii) From (i) and (ii), we obtain the desired results (F1), (F2), (F3) for any $k(\geq 2)$.

Step 4. We have proved (1) and (2) of the present proposition in the case that $\left(\theta_{0}, \theta_{\infty}\right) \in \Sigma_{-}$. By Proposition 3.8, we can also obtain the same result in the case that $\left(\theta_{0}, \theta_{\infty}\right) \in \Sigma_{+}$. Hence we have finished the proof.

\subsection{Rational solutions of $P_{I I I}\left(\theta_{0}, \theta_{\infty}\right)$ and proof of Theorem 2}

Using the results in previous subsections, we give a proof of Theorem 2 . We 
prove the following proposition first, and then prove Theorem 2 .

Proposition 3.11. (1) $P_{\text {III }}\left(\theta_{0}, \theta_{\infty}\right)$ does not have algebraic solutions.

(2) $P_{\mathrm{III}}\left(\theta_{0}, \theta_{\infty}\right)$ has rational solutions if and only if there exists an integer $I$ such that $\theta_{\infty}-\theta_{0}-1=2 I$ or $\theta_{\infty}+\theta_{0}+1=2 I$, where $\left(\theta_{0}, \theta_{\infty}\right) \in \mathbf{C}^{2}$.

(3) If $P_{\mathrm{III}}\left(\theta_{0}, \theta_{\infty}\right)$ has rational solutions, then the number of rational solutions is two or four. $P_{\mathrm{III}}\left(\theta_{0}, \theta_{\infty}\right)$ has four rational solutions if and only if there exist two integers $I$ and $J$ such that $\theta_{\infty}-\theta_{0}-1=2 I$ and $\theta_{\infty}+\theta_{0}+1=2 J$.

(4) Rational solutions are classified as in the table IV. Here the sets $D_{ \pm}, \Delta_{ \pm}$and the numbers $\alpha_{ \pm}$are the same ones as in Theorem 2, and double signs correspond with each other. In the types $\mathrm{III}_{ \pm}, \mathrm{IV}_{ \pm}$, the values of $N, \varepsilon_{j}, b_{j}(j=1, \cdots, N)$ depend on the values of $\theta_{0}, \theta_{\infty}$ and $\alpha_{ \pm}$.

\begin{tabular}{|c|c|c|}
\hline Type & Conditions of $\left(\theta_{0}, \theta_{\infty}\right)$ & Forms of rational solutions \\
\hline $\mathrm{I}_{ \pm}$ & $\theta_{\infty} \mp \theta_{0} \mp 1=\left(\theta_{0}, \theta_{\infty}\right) \in \mathbf{C}^{2}$ & $y=\alpha_{ \pm}, \quad y=-\alpha_{ \pm}$ \\
\hline $\mathrm{II}_{ \pm}$ & $\left(\theta_{0}, \theta_{\infty}\right) \in D_{ \pm}$ & $y=a_{ \pm}+\theta_{\infty} / x, \quad y=-\alpha_{ \pm}+\theta_{\infty} / x$ \\
\hline $\mathrm{III}_{ \pm}$ & $\begin{array}{l}\theta_{\infty} \mp \theta_{0} \mp 1=2 I,\left(\theta_{0}, \theta_{\infty}\right) \in \mathbf{C}^{2} \\
I \in \mathbf{Z}-\{0\},\left(\theta_{0}, \theta_{\infty}\right) \notin D_{ \pm} \cup \Delta_{ \pm}\end{array}$ & $\begin{array}{c}y=\alpha_{ \pm}+\frac{1}{2} \sum_{j=1}^{N} \frac{\varepsilon_{j}}{x-b_{j}} \\
y=-\alpha_{ \pm}+\frac{1}{2} \sum_{j=1}^{N} \frac{\varepsilon_{j}}{x+b_{j}} \\
\left(\begin{array}{l}N, \varepsilon_{j}, b_{j}, I \text { satisfy } \\
\text { the same conditions } \\
\text { as in Theorem } 2, \mathrm{III}_{ \pm}(*)\end{array}\right) \cdots(* *)\end{array}$ \\
\hline $\mathrm{IV}_{ \pm}$ & $\left(\theta_{0}, \theta_{\infty}\right) \in \Delta_{ \pm}$ & $\begin{array}{c}\qquad y=\alpha_{ \pm}+\frac{\theta_{\infty}}{x}+\frac{1}{2} \sum_{j=1}^{N} \frac{\varepsilon_{j}}{x-b_{j}} \\
y=-\alpha_{ \pm}+\frac{\theta_{\infty}}{x}+\frac{1}{2} \sum_{j=1}^{N} \frac{\varepsilon_{j}}{x+b_{j}} \\
\text { under the same conditions as in (**) }\end{array}$ \\
\hline
\end{tabular}

Table IV. Rational solutions of $P_{\mathrm{III}}\left(\theta_{0}, \theta_{\infty}\right)$.

Proof. (1) If $y(x)$ is a $k$-sheeted $(k \geq 1)$ algebraic solution of $P_{\mathrm{III}}\left(\theta_{0}, \theta_{\infty}\right)$, then $q(t)=\sqrt{t} y(\sqrt{t})$ is an algebraic solution of $P_{\mathrm{III}}\left(\theta_{0}, \theta_{\infty}\right)$. Since $q\left(x^{2}\right) / x=$ $\sqrt{x^{2}} y\left(\sqrt{x^{2}}\right) / x=y(x)$ is a rational solution by Proposition 3.2 , it turns out that $k=1$. Therefore, $P_{\text {III }}\left(\theta_{0}, \theta_{\infty}\right)$ does not have a many-valued algebraic solution.

(2) (3) (4) From Propositions 3.5, 3.6 and 3.10, we obtain the results for rational 
solutions of the type $J_{+}\left(J_{+}=\mathrm{I}_{+}, \cdots, \mathrm{IV}_{+}\right)$. Next, from these results and Proposition 3.4 , we obtain the results for rational solutions of the type $J_{-}\left(J_{-}=\mathrm{I}_{-}, \cdots\right.$, IV_). By Proposition 3.3, we thus exhausted all rational solutions of $P_{\mathrm{III}}\left(\theta_{0}, \theta_{\infty}\right)$.

Remark 3.3. Gromak's result [5, Theorem 9] corresponds to (2) in this proposition.

Proof of Theorem 2. From Propositions 3.2 and Proposition 3.11, we obtain the desired results. Here, by the following calculation, we can check that two rational solutions of the type $J_{ \pm}(J=\mathrm{I}, \cdots, \mathrm{IV})$ give the same algebraic solution of $P_{\mathrm{III}}\left(\theta_{0}, \theta_{\infty}\right)$. For example, we assume $J_{+}=\mathrm{IV}_{+}$, and take

$$
\begin{aligned}
& y_{1}(x)=1+\theta_{\infty} / x+(1 / 2) \sum_{j=1}^{N} \varepsilon_{j} /\left(x-b_{j}\right) \\
& y_{2}(x)=-1+\theta_{\infty} / x+(1 / 2) \sum_{j=1}^{N} \varepsilon_{j} /\left(x+b_{j}\right) .
\end{aligned}
$$

Since

$$
\begin{aligned}
& q_{1}(t)=\sqrt{t} y_{1}(\sqrt{t})=\sqrt{t}\left\{1+\theta_{\infty} / \sqrt{t}+(1 / 2) \sum_{j=1}^{N} \varepsilon_{j} /\left(\sqrt{t}-b_{j}\right)\right\} \\
& q_{2}(t)=\sqrt{t} y_{2}(\sqrt{t})=\sqrt{t}\left\{-1+\theta_{\infty} / \sqrt{t}+(1 / 2) \sum_{j=1}^{N} \varepsilon_{j} /\left(\sqrt{t}+b_{j}\right)\right\},
\end{aligned}
$$

if we analytically continue $q_{1}(t)$ around $t=0$,

$$
q_{1}(t)=(-\sqrt{t})\left\{1+\theta_{\infty} /(-\sqrt{t})+(1 / 2) \sum_{j=1}^{N} \varepsilon_{j} /\left(-\sqrt{t}-b_{j}\right)\right\}=q_{2}(t) .
$$

\section{Proof of Theorem 3}

In this section, we suppose that parameters $\theta_{0}$ and $\theta_{\infty}$ of $P_{\mathrm{III}}\left(\theta_{0}, \theta_{\infty}\right)$ always satisfy the condition $\theta_{\infty}+\theta_{0}=2 I$ or $\theta_{\infty}-\theta_{0}=2 J$, where $I$ and $J$ are any integers. To clarify the condition which parameters $\theta_{0}$ and $\theta_{\infty}$ fulfill, we will often use notations $P_{\mathrm{III}}\left(\theta_{\infty}+\theta_{0}=2 I\right), P_{\mathrm{III}}\left(\theta_{\infty}-\theta_{0}=2 J\right)$ (or $P_{\mathrm{III}}\left(\theta_{\infty}+\theta_{0}+1=2 I+1\right)$, $\left.P_{\mathrm{III}}\left(\theta_{\infty}-\theta_{0}-1=2 J-1\right)\right)$. Let us consider the following four Riccati equations:

$$
\begin{aligned}
& R_{1}: d q / d t=(1 / t)\left(-q^{2}-\theta_{0} q+t\right) \\
& R_{-1}: d q / d t=(1 / t)\left(q^{2}-\theta_{\infty} q-t\right) \\
& \bar{R}_{1}: d q / d t=(1 / t)\left(-q^{2}+\theta_{\infty} q-t\right) \\
& \bar{R}_{-1}: d q / d t=(1 / t)\left(q^{2}-\theta_{0} q+t\right)
\end{aligned}
$$

$R_{1}$ coincides with the equation (1.9) and is contained in $P_{\mathrm{III}}\left(\theta_{\infty}+\theta_{0}+1=1\right)$. $R_{-1}, \bar{R}_{1}$ and $\bar{R}_{-1}$ are contained in $P_{\mathrm{III}}\left(\theta_{\infty}+\theta_{0}+1=-1\right), P_{\mathrm{III}}\left(\theta_{\infty}-\theta_{0}-1=1\right)$ 
and $P_{\mathrm{III}}\left(\theta_{\infty}-\theta_{0}-1=-1\right)$ respectively. We express the Riccati equation $R_{1}$ contained in $P_{\mathrm{III}}\left(\theta_{\infty}+\theta_{0}+1=1\right)$ by $R_{1}\left(\theta_{\infty}+\theta_{0}+1=1\right)$. Similarly we use notations $R_{-1}\left(\theta_{\infty}+\theta_{0}+1=-1\right), \bar{R}_{1}\left(\theta_{\infty}-\theta_{0}-1=1\right)$ and $\bar{R}_{-1}\left(\theta_{\infty}-\theta_{0}\right.$ $-1=-1$ ). If we apply the transformation $h$ to solutions of $R_{1}$, then we get solutions of $R_{-1}$. Conversely, if we apply $h$ to solutions of $R_{-1}$, then we get solutions of $R_{1}$. We express this relation by the symbol

$$
R_{1} \stackrel{h}{\longrightarrow} R_{-1} \text {. }
$$

In the same meanings, we have the following relations:

$$
\begin{aligned}
& \bar{R}_{1} \stackrel{\leftrightarrow}{\longleftrightarrow} \bar{R}_{-1} \\
& R_{1} \stackrel{s_{2}}{\longleftrightarrow} \bar{R}_{-1}, \quad R_{-1} \stackrel{s_{2}}{\longrightarrow} \bar{R}_{1}
\end{aligned}
$$

Proposition 4.1. Transformation $l^{ \pm 1}$ (resp. $m^{ \pm 1}$ ) is applicable to a solution $q(t)$ of $P_{\mathrm{III}}\left(\theta_{0}, \theta_{\infty}\right)$ if and only if $q(t)$ is not a solution of $R_{\mp}\left(\right.$ resp. $\left.\bar{R}_{ \pm 1}\right)$. Here double signs correspond with each other, and $R_{+1}, \bar{R}_{+1}$ denote $R_{1}, \bar{R}_{1}$ respectively.

Proof. We prove only the case of $l$. Let $q(t)$ be a solution of $P_{\mathrm{III}^{\prime}}\left(\theta_{0}, \theta_{\infty}\right)$. We can not apply $l$ to $q(t)$ if and only if $q(t)$ satisfies the equation

$$
d q / d t=(1 / t)\left(q^{2}-A q-t\right)
$$

where $A=-\left(\theta_{0}+2\right)$ or $A=\theta_{\infty}$.

Suppose $q(t)$ satisfies (4.3). Differentiating (4.3) by $t$, we obtain

$$
\frac{d^{2} q}{d t^{2}}=\frac{2 q^{3}-(3 A+1) q^{2}+\left(-2 t+A^{2}+A\right) q+A t}{t^{2}} .
$$

Substituting (4.3) and (4.4) into

$$
t^{2} q \frac{d^{2} q}{d t^{2}}=\left(t \frac{d q}{d t}\right)^{2}-t q \frac{d q}{d t}+q^{3}\left(q-\theta_{\infty}\right)+\left(\theta_{0}+1\right) t q-t^{2}
$$

we obtain

$$
\left(\theta_{\infty}-A\right) q^{2}-\left(\theta_{0}+2+A\right) t=0 .
$$

Whichever $A$ may be, (4.5) induces $\theta_{\infty}+\theta_{0}=-2$, and (4.3) coincides with $R_{-1}\left(\theta_{\infty}+\theta_{0}+1=-1\right)$. Conversely, if $q(t)$ is a solution of $R_{-1}\left(\theta_{\infty}+\theta_{0}+1=\right.$ $-1)$, then $q(t)$ satisfies (4.3). 
We put

$$
\begin{aligned}
& \Pi_{ \pm 1}=\left\{\left(\theta_{0}, \theta_{\infty}\right) \in \mathbf{C}^{2} \mid \theta_{\infty}+\theta_{0}+1= \pm 1\right\} \\
& \bar{\Pi}_{ \pm 1}=\left\{\left(\theta_{0}, \theta_{\infty}\right) \in \mathbf{C}^{2} \mid \theta_{\infty}-\theta_{0}-1= \pm 1\right\} .
\end{aligned}
$$

Apparently, we have

$$
\begin{array}{ll}
\Pi_{1} \cap \bar{\Pi}_{1}=\{(-1,1)\} & \Pi_{1} \cap \bar{\Pi}_{-1}=\{(0,0)\}, \\
\Pi_{-1} \cap \bar{\Pi}_{1}=\{(-2,0)\}, & \Pi_{-1} \cap \bar{\Pi}_{-1}=\{(-1,-1)\} .
\end{array}
$$

In connection with these facts, we have the following proposition.

Proposition 4.2. $\quad P_{\mathrm{III}^{\prime}}(-1,1)$ contains two Riccati equations $R_{1}, \bar{R}_{1}$ and these equations do not have common solutions. Similar results hold for $P_{\mathrm{III}^{\prime}}(0,0)$ and $\left(R_{1}\right.$, $\left.\bar{R}_{-1}\right), P_{\mathrm{III}}(-2,0)$ and $\left(R_{-1}, \bar{R}_{1}\right), P_{\mathrm{III}}(-1,-1)$ and $\left(R_{-1}, \bar{R}_{1}\right)$ respectively.

We omit the proof. By simple calculations, we obtain the following result.

PROPOSITION 4.3. (1) If $q(t)$ is a solution of $R_{ \pm 1}\left(\theta_{\infty}+\theta_{0}+1= \pm 1\right)$, then $m(q(t))$ (resp. $\left.m^{-1}(q(t))\right)$ is a solution of $R_{ \pm 1}\left(\left(\theta_{\infty}-1\right)+\left(\theta_{0}+1\right)+1= \pm 1\right)$ (resp. $R_{ \pm 1}\left(\left(\theta_{\infty}+1\right)+\left(\theta_{0}-1\right)+1= \pm 1\right)$ ).

(2) If $q(t)$ is a solution of $\bar{R}_{ \pm 1}\left(\theta_{\infty}-\theta_{0}-1= \pm 1\right)$, then $l(q(t))$ (resp. $l^{-1}(q(t))$ ) is a solution of $\bar{R}_{ \pm 1}\left(\left(\theta_{\infty}+1\right)-\left(\theta_{0}+1\right)-1= \pm 1\right)\left(\right.$ resp. $\bar{R}_{ \pm 1}\left(\left(\theta_{\infty}-1\right)-\right.$ $\left.\left.\left(\theta_{0}-1\right)-1= \pm 1\right)\right)$.

Using the above results, we can prove Theorem 3 .

Proof of Theorem 3. Let $I$ be any integer, $t$ be the independent variable of $P_{\mathrm{III}}\left(\theta_{\infty}+\theta_{0}=2 I\right)$. Let $\phi_{\sigma}(t)$ (resp. $\left.\phi_{\sigma}(t)\right)(\sigma \in \mathbf{C})$ be a general solution of $R_{1}\left(\theta_{\infty}+\theta_{0}=0\right)$ (resp. $R_{-1}\left(\theta_{\infty}+\theta_{0}=-2\right)$ ). By Proposition 4.1, $l^{I}\left(\phi_{\sigma}(t)\right)$ $(\sigma \in \mathbf{C})$ is a one-parameter family of solutions of $P_{\mathrm{III}}\left(\bar{\theta}_{\infty}+\bar{\theta}_{0}=2 I\right)$, where $I \geq 0$, $\bar{\theta}_{0}=\theta_{0}+I, \bar{\theta}_{\infty}=\theta_{\infty}+I$. When $I=1, l\left(\phi_{\sigma}(t)\right)$ and $(d / d t)\left\{l\left(\phi_{\sigma}(t)\right)\right\}$ are rational functions of $\bar{\theta}_{0}, t$ and $\phi_{\sigma}(t)$ with integer coefficients. By induction, we find that $l^{I}\left(\phi_{\sigma}(t)\right)$ is a rational function of $\bar{\theta}_{0}, t$ and $\phi_{\sigma}(t)$ with integer coefficients. By similar arguments, we see that $P_{\mathrm{III}}\left(\bar{\theta}_{\infty}+\bar{\theta}_{0}=-2(I+1)\right)$ has a one-parameter family of solutions $\left(l^{-1}\right)^{I}\left(\phi_{\sigma}(t)\right)$, which is a rational function of $\bar{\theta}_{0}, t$ and $\phi_{\sigma}(t)$ with integer coefficients, where $I \geq 0, \bar{\theta}_{0}=\theta_{0}-I, \bar{\theta}_{\infty}-I$. Here let us recall the correspondence of $R_{1}$ and $R_{-1}$ by the transformation $h$ (See (4.1)). By this correspondence, we see that $P_{\mathrm{III}}\left(\bar{\theta}_{0}+\bar{\theta}_{\infty}=2 I\right)$ has a one-parameter family of solutions of the form 


$$
q(t)=\mathscr{Q}_{I}\left(\bar{\theta}_{0}, t, \phi_{\sigma}(t)\right),
$$

where $\mathscr{Q}_{I}$ is a rational function of three variables with integer coefficients. The form of $\mathscr{Q}_{I}$ depends on the value of $I$. From (4.2) and the same arguments as in the above, we find that $P_{\mathrm{III}}\left(\bar{\theta}_{\infty}-\bar{\theta}_{0}=-2 J\right)$ has a one-parameter family of solution of the form

$$
\bar{q}\left(t_{1}\right)=\overline{\mathscr{Q}}_{-J}\left(\bar{\theta}_{0}, t_{1}, \phi_{\sigma}\left(-t_{1}\right)\right),
$$

where $J$ is any integer, $t_{1}=-t$ is the independent variable of $P_{\mathrm{III}}\left(\bar{\theta}_{\infty}-\bar{\theta}_{0}=\right.$ $-2 J), \bar{Q}_{-J}$ has the same property as $\mathscr{Q}_{I}$ in (4.6).

Next, we suppose that $\left(\bar{\theta}_{0}, \bar{\theta}_{\infty}\right)$ satisfy the conditions $\bar{\theta}_{\infty}+\bar{\theta}_{0}=2 I$ and $\bar{\theta}_{\infty}-$ $\bar{\theta}_{0}=-2 J$, where $I, J$ are nonnegative integers. We use a variable $t$ as the independent variable of $P_{\mathrm{III}}\left(\bar{\theta}_{0}, \bar{\theta}_{\infty}\right)$. Then, $P_{\mathrm{III}}\left(\bar{\theta}_{0}, \bar{\theta}_{\infty}\right)$ has a one-parameter family of solutions $q(t)$ of the form (4.6) and a one-parameter family of solutions $\bar{q}(t)$ of the form (4.7). Noting Proposition 4.3 and the fact that transformations $l$ and $m$ do not change the independent variable of $P_{\mathrm{III}^{\prime}}\left(\theta_{0}, \theta_{\infty}\right)$, and that $l m=m l$, we see that

$$
\begin{aligned}
q(t) & =l^{I}\left(\phi_{\sigma}\left(t ; \theta_{\infty}+\theta_{0}=0\right)\right)=l^{I}\left(m^{J}\left(\phi_{\sigma}(t ; 0+0=0)\right)\right) \\
& =m^{J}\left(l^{I}\left(\phi_{\sigma}(t ; 0+0=0)\right)\right), \\
\bar{q}(t) & =m^{J}\left(\bar{\phi}_{\sigma}\left(t ; \theta_{\infty}-\theta_{0}=0\right)\right)=m^{J}\left(l^{I}\left(\bar{\phi}_{\sigma}(t ; 0-0=0)\right)\right),
\end{aligned}
$$

where $\phi_{\sigma}\left(t ; \theta_{\infty}+\theta_{0}=0\right), \bar{\phi}_{\sigma}\left(t ; \theta_{\infty}-\theta_{0}=0\right)$ are general solutions of the Riccati equations $R_{1}\left(\theta_{\infty}+\theta_{0}=0\right), \bar{R}_{-1}\left(\theta_{\infty}-\theta_{0}=0\right)$ respectively (Refer to Figure 2). By Proposition $4.2, R_{1}(0+0=0), \bar{R}_{-1}(0-0=0)$ do not have common solutions. Therefore one-parameter families $q(t)$ and $\bar{q}(t)$ can not have common solutions. When $I$ and $J$ satisfy other conditions, for example, $\mathrm{I} \leq-1, J \geq 0$, we can prove in the same way. 


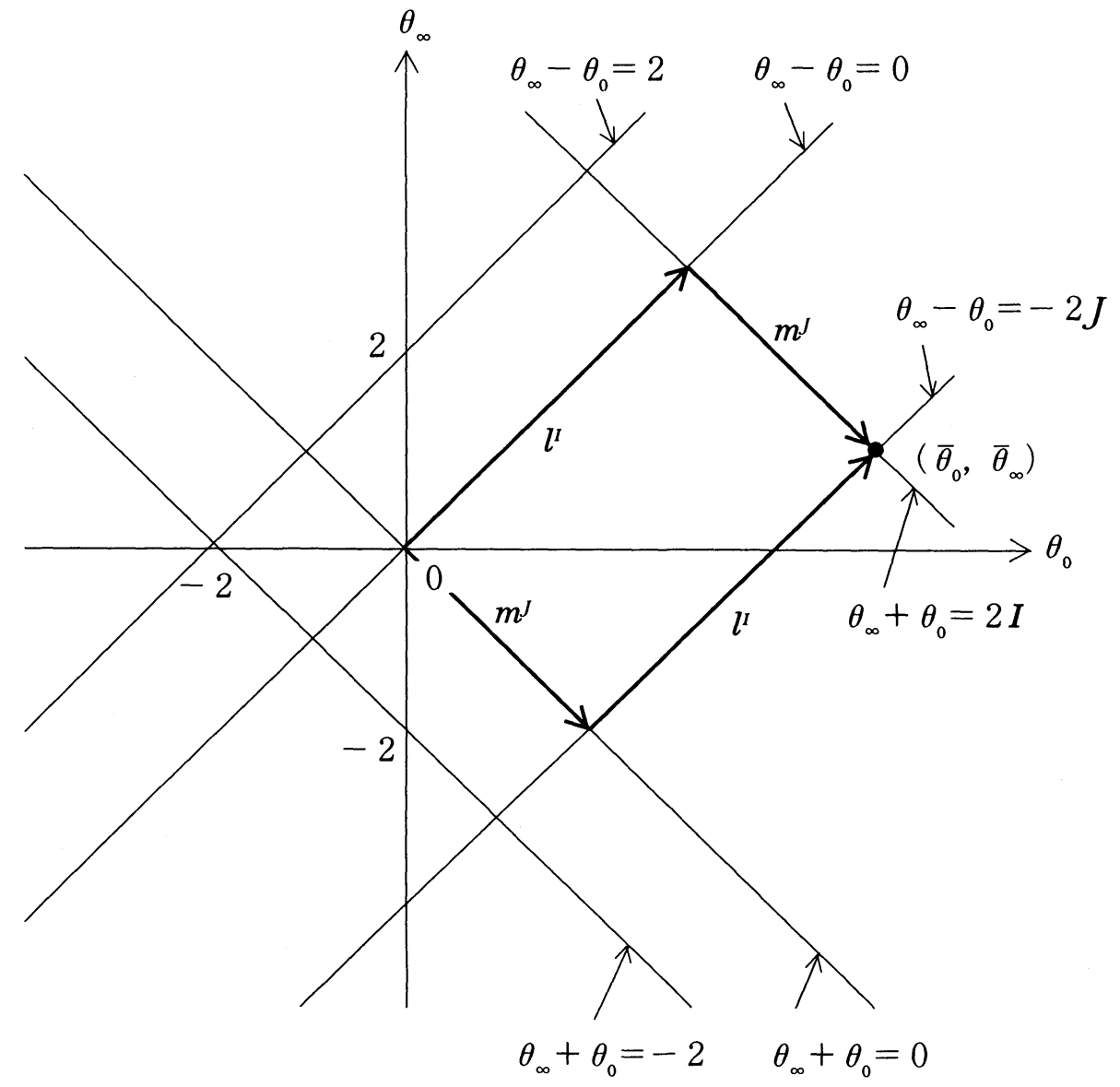

Figure 2. The relation $m^{J} l^{I}=l^{I} m^{J}$ holds.

\section{REFERENCES}

[1] V. I. Gromak, Solutions of the third Painlevé equation, Differential Equations, 9 (1973), 1599-1600.

[2] - Theory of Painlevé's equation, Differential Equations, 11 (1975), 285-287.

[3] - One-parameter systems of solutions of Painlevé's equations, Differential Equations, 14 (1978), 1510-1513.

[4] V. I. Gromak and N. A. Lukashevich, Special classes of solutions of Painlevé's equations, Differential Equations, 18 (1982), 317-326.

[5] V. I. Gromak, Reducibility of Painlevé equations, Differential Equations, 20 (1984), $1191-1198$.

[6] N. A. Lukashevich, On the theory of the third Painlevé equation, Differential Equations, 3 (1967), 994-999. 
[7] Y. Murata, Rational solutions of the second and the fourth Painlevé equations, Funkcial. Ekvac., 28 (1985), 1-32.

[8] - On the irreducibility of the third Painleve equation, in preparation.

[9] K. Nishioka, A note on the transcendency of Painlevé's first transcendent, Nagoya Math. J., 109 (1988), 63-67.

[10] M. Noumi, private communication.

[11] K. Okamoto, Polynomial Hamiltonians associated with Painlevé equations. I, Proc. Japan Acad. Ser. A Math. Sci., 56 (1980), 264-268; II, ibid., 367-371.

[12] - Isomonodromic deformation and Painlevé equations, and the Garnier system, J. Fac. Sci. Univ. Tokyo Sect. IA Math., 33 (1986), 575-618.

[13] - Studies on the Painlevé equations IV. Third Painlevé equation $P_{\text {III }}$, Funkcial. Ekvac., 30 (1987), 305-332.

[14] - private communication.

[15] P. Painlavé, Leçons de Stockholm, in "EEuvres de P. Painlevé I", Editions du C. N. R. S., Paris, 1972, 199-818.

[16] H. Umemura, Birational automorphism groups and differential equations, Nagoya Math. J., 119 (1990), 1-80.

[17] — On the irreducibility of the first differential equation of Painlevé, in "Alge braic geometry and commutative algebra in honor of Masayoshi Nagata", Kinokuniya, Tokyo, 1987, 771-789.

Faculty of Economics

Nagasaki University

4-2-1, Katafuchi, Nagasaki-shi 850

Japan 